\title{
Some Dirichlet spaces obtained by subordinate reflected diffusions
}

Niels Jacob and René L. Schilling

In this paper we want to show how well-known results from the theory of (regular) elliptic boundary value problems, function spaces and interpolation, subordination in the sense of Bochner, and Dirichlet forms can be combined and how one can thus get some new aspects in each of these fields.

Let $A=L(x, D)$ be a second-order elliptic differential operator with smooth coefficients on a bounded domain $G$ with smooth boundary $\partial G$ and with Dirichlet or Neumann boundary conditions. Assume that the operator is symmetric. Under Neumann boundary conditions, it generates a reflected diffusion process $\left\{X_{t}\right\}_{t \geq 0}$ which is associated with a Dirichlet form $\mathcal{E}$ with domain $H^{1}(G)$. It is clear that $A$ defined on

$$
D(A)=H_{\{\partial / \partial \nu\}}^{2}(G):=\left\{u \in H^{2}(G):\left.\frac{\partial}{\partial \nu} u\right|_{\partial G}=0\right\}
$$

is also the generator of a sub-Markovian semigroup $\left\{T_{t}\right\}_{t \geq 0}$ on $L^{2}(\bar{G})$. Denote by $f_{\alpha}, 0<\alpha<1$, the Bernstein function $f_{\alpha}(x)=x^{\alpha}$. By subordination in the sense of Bochner it is possible to construct for each $\alpha \in(0,1)$ four new objects, $A^{(\alpha)}:=-(-A)^{\alpha},\left\{T_{t}^{(\alpha)}\right\}_{t \geq 0}$, the semigroup generated by $A^{(\alpha)}, \mathcal{E}^{(\alpha)}(\cdot, \cdot)$, the Dirichlet form associated with $A^{(\alpha)}$ (and also with $\left\{T_{t}^{(\alpha)}\right\}_{t \geq 0}$ ), and the subordinate (with respect to the Bernstein function $\left.x^{\alpha}\right)$ stochastic process $\left\{X_{t}^{(\alpha)}\right\}_{t \geq 0}$. These constructions are of a somewhat abstract nature and some work has to be done 
if one wants to determine $D\left(A^{(\alpha)}\right)$ and $D\left(\mathcal{E}^{(\alpha)}\right)$ explicitly in terms of function spaces. In fact, this work has already been done by R. Seeley [18] for $D\left(A^{(\alpha)}\right)$, and for $D\left(\mathcal{E}^{(\alpha)}\right)$ the results are even longer known, $c f$. J. L. Lions and E. Magenes [13], and, as reference for both cases, the monograph [22] by H. Triebel.

In our first section we collect some fundamental results on the Dirichlet and Neumann problems for second-order elliptic differential operators (with smooth coefficients in a domain with smooth boundary) and the associated diffusion processes. Subordination in the sense of Bochner will be discussed in Section 2, both from the analytic and probabilistic point of view. In the third section we study $D\left(A^{(\alpha)}\right)$ and $D\left(\mathcal{E}^{(\alpha)}\right)$ under Dirichlet and Neumann conditions. In both cases the domains are certain fractional order Sobolev spaces. Under Neumann boundary conditions we have

$$
D\left(\mathcal{E}^{(\alpha)}\right)=H^{\alpha}(G), \quad \text { if } \alpha \in(0,1)
$$

and

$$
\begin{gathered}
D\left(A^{(\alpha)}\right)=H_{\{\partial / \partial \nu\}}^{2 \alpha}(G), \quad \text { if } \alpha \in\left(\frac{3}{4}, 1\right), \\
D\left(A^{(\alpha)}\right)=H^{2 \alpha}(G), \quad \text { if } \alpha \in\left(0, \frac{3}{4}\right),
\end{gathered}
$$

under Dirichlet boundary conditions we have

$$
\begin{aligned}
& D\left(\mathcal{E}^{(\alpha)}\right)=H^{\alpha}(G), \quad \text { if } \alpha \in\left(0, \frac{1}{2}\right), \\
& D\left(\mathcal{E}^{(\alpha)}\right)=H_{0}^{\alpha}(G), \quad \text { if } \alpha \in\left(\frac{1}{2}, 1\right),
\end{aligned}
$$

and

$$
\begin{array}{ll}
D\left(A^{(\alpha)}\right)=H^{2 \alpha}(G), & \text { if } \alpha \in\left(0, \frac{1}{4}\right), \\
D\left(A^{(\alpha)}\right)=H_{D}^{2 \alpha}(G), & \text { if } \alpha \in\left(\frac{1}{4}, 1\right) .
\end{array}
$$

Here,

$$
H_{D}^{s}(G):=\left\{u \in H^{s}(G): \gamma u=0\right\}
$$


with the trace operator $\gamma$. One should note that these are well-known results in the theory of elliptic boundary value problems, but they seem to be rather ignored in the theory of Dirichlet forms.

Section 4 deals with the decomposition of the (Neumann) Dirichlet space $\left(\mathcal{E}_{\lambda}^{(\alpha)}, H^{\alpha}(G)\right)$. We show that $H^{\alpha}(G)$ can be written as an orthogonal sum $H_{0}^{\alpha}(G) \oplus \mathcal{H}_{\lambda}^{\alpha}(G)$ where the functions $u \in \mathcal{H}_{\lambda}^{\alpha}(G)$ are the harmonic functions with respect to the form $\mathcal{E}_{\lambda}^{(\alpha)}$ - i.e. $\mathcal{E}_{\lambda}^{(\alpha)}(u, w)=0$ for all $w \in H_{0}^{\alpha}(G)$. Moreover, we show that there is an isomorphism $\Pi_{\lambda}^{(\alpha)}$ from $H^{\alpha-1 / 2}(\partial G)$ to $\mathcal{H}_{\lambda}^{\alpha}(G)$. This map establishes a unitary equivalence between $\left(\mathcal{E}_{\lambda}^{(\alpha)}, \mathcal{H}^{\alpha}(G)\right)$ and $\left(\mathcal{C}_{\lambda}^{(\alpha)}, H^{\alpha-1 / 2}(\partial G)\right)$, where $\mathcal{C}_{\lambda}^{(\alpha)}$ is at least for $\lambda=0$ - the analogue of the classical Douglas integral. This correspondence is further investigated in Section 5. In particular, we show that $\left(\mathcal{C}_{\lambda}^{(\alpha)}, H^{\alpha-1 / 2}(\partial G)\right)$ is a regular Dirichlet space and that $\mathcal{C}_{\lambda}^{(\alpha)}$ is equivalent to the canonical scalar product on $H^{\alpha-1 / 2}(\partial G)$ which itself is a Dirichlet form. The precise knowledge of $D\left(\mathcal{C}_{\lambda}^{(\alpha)}\right)$ allows us,

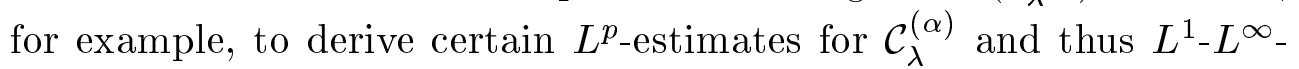
estimates for the associated semigroup.

In Section 6 we construct the associated boundary processes and show that the process generated by $\left(\mathcal{C}_{\lambda}^{(\alpha)}, H^{\alpha-1 / 2}(\partial G)\right)$ can indeed be obtaind by an appropriate time-change of the process generated by $\left(\mathcal{C}_{\lambda}, H^{1 / 2}(\partial G)\right)$.

The final section takes up the Skorokhod representation of the reflected diffusion which was already discussed in the first section. We use now Bochner's subordination (with respect to fractional powers) in order to derive a representation for the subordinate reflected process. Note, that subordination is one possibility to construct a reflected symmetric stable process in a unique and natural way. However, in [24] S. Watanabe pointed out that there are several methods of getting processes which one could call reflected symmetric stable processes.

\section{Dirichlet forms generated by elliptic differential operators with boundary conditions.}

In this section we summarize some results on Dirichlet forms that are generated by second-order elliptic differential operators satisfying Neumann or Dirichlet boundary conditions. In particular, we recall some conditions that allow to associate stochastic processes to these Dirichlet forms, reflected diffusions under Neumann boundary condi- 
tions and absorbing diffusions under Dirichlet boundary conditions. Since we want to present our ideas as clearly as possible (and do not want to get entangled in technical details) we will restrict our considerations to rather smooth objects - thus getting at best sub-optimal conditions from the point of view of Dirichlet forms, but keeping full compatibility with existing (analytic) literature. Our exposition will, later on, rely heavily on results from the theory of function spaces and interpolation theory.

The main reference for this section is the monograph [8] by M. Fukushima, Y. Oshima, and M. Takeda. For the Neumann problem we refer especially to the paper [9] by M. Fukushima and M. Tomisaki. We should, however, mention that the crux of that paper was to consider a situation with rather weak regularity assumptions - which is somehow an opposite point of view. Nevertheless we think it might be convenient for the reader to have a state-of-the-art and easily accessible reference.

Let $G \subset \mathbb{R}^{n}$ be a bounded domain with smooth boundary $\partial G$, i.e., $\partial G$ is assumed to be a $C^{\infty}$-manifold. We consider the second order differential operator

$$
L(x, D)=\sum_{k, \ell=1}^{n} \frac{\partial}{\partial x_{k}}\left(a_{k \ell}(x) \frac{\partial}{\partial x_{\ell}}\right),
$$

with coefficients $a_{k \ell}=a_{\ell k} \in C^{\infty}(\bar{G})$. Moreover, we assume that

$$
\lambda_{0}^{-1}|\xi|^{2} \leq \sum_{k, \ell=1}^{n} a_{k \ell}(x) \xi_{k} \xi_{\ell} \leq \lambda_{0}|\xi|^{2}
$$

for some $\lambda_{0}>0$ and all $x \in \bar{G}, \xi \in \mathbb{R}^{n}$. It is well known that the quadratic form

$$
\mathcal{E}(u, v):=\int_{G} \sum_{k, \ell=1}^{n} a_{k \ell}(x) \frac{\partial u}{\partial x_{k}}(x) \frac{\partial v}{\partial x_{\ell}}(x) d x,
$$

with domain $H^{1}(G) \subset L^{2}(\bar{G})$ is a regular Dirichlet form, see [8, Example 1.6.1], where the regularity problem is carefully discussed if $a_{k \ell}(x)=\delta_{k \ell}$. Therefore, $c f$. [9], there exists a conservative diffusion process $\mathbf{X}=\left(\left\{X_{t}\right\}_{t \geq 0}, \mathbb{P}^{x},\left\{\mathfrak{F}_{t}\right\}_{t \geq 0}\right)$ on $\bar{G}$ which is associated with the Dirichlet form (1.3). For each $t \geq 0$ and $x \in \bar{G}$ the transition function $p_{t}(x, \cdot)$ of $\mathbf{X}$ is known to be absolutely continuous with respect to Lebesgue measure, and $\mathbf{X}$ is a strong Feller process. 
Due to our regularity assumptions, the domain $D(A)$ of the generator $A$ of the Dirichlet form $\mathcal{E}$ is given by

$$
D(A)=\left\{u \in H^{2}(G): \frac{\partial}{\partial \nu} u=0\right\},
$$

where $\partial / \partial \nu$ denotes the derivative in direction of the outer normal $\nu=\left(\nu_{1}, \ldots, \nu_{n}\right)$ to the boundary $\partial G$. Sometimes we will also write

$$
H_{\{\partial / \partial \nu\}}^{2}(G)=D(A) \text {. }
$$

On $D(A)$ we have $A=L(x, D)$ which can be interpreted to hold in strong $L^{2}$-sense, but, of course, also in the sense of distributions. Let us observe for later applications that $(L(x, D), \partial / \partial \nu)$ forms a regular elliptic boundary value problem in the sense of S. Agmon, A. Douglis, and L. Nirenberg [1], see also [22] which will be our standard reference.

The general theory of Dirichlet forms shows that we can always associate a sub-Markovian semigroup $\left\{T_{t}\right\}_{t \geq 0}$ on $L^{2}(\bar{G})$ with $\left(\mathcal{E}, H^{1}(G)\right)$. In our case, this semigroup enjoys the strong Feller property, it is conservative, i.e. $T_{t} 1=1$, and its transition kernels have densities with respect to Lebesgue measure on $\bar{G}$, i.e., we have

$$
T_{t} u(x)=\mathbb{E}^{x}\left(u\left(X_{t}\right)\right)=\int_{\bar{G}} p_{t}(x, y) u(y) d y .
$$

We call $\left\{T_{t}\right\}_{t \geq 0}$ the Neumann semigroup associated with the Dirichlet form $\left(\mathcal{E}, H^{1}(\bar{G})\right)$.

One of the major aims in [9] was to obtain a Skorokhod representation of the process $\mathbf{X}$ under minimal smoothness conditions. Of course, this result remains valid in the situation considered here and reads as follows: Let $X_{t}^{k}$ denote the $k$-th coordinate of $X_{t}, 1 \leq k \leq n$. For $t \geq 0$ and $x \in \bar{G}$ one has almost surely $\left(\mathbb{P}^{x}\right)$

$$
\begin{aligned}
X_{t}^{k}-X_{0}^{k}= & M_{t}^{k}+\sum_{\ell=1}^{n} \int_{0}^{t} \frac{\partial a_{k \ell}}{\partial x_{\ell}}\left(X_{s}\right) d s \\
& +\sum_{\ell=1}^{n} \int_{0}^{t} a_{k \ell}\left(X_{s}\right) \nu_{\ell}\left(X_{s}\right) d L_{s} .
\end{aligned}
$$

Here, $M_{t}^{k}, 1 \leq k \leq n$, are continuous additive functionals in the strict sense, see [8, p. 181, p. 326] for the definition, satisfying

$$
\mathbb{E}^{x}\left(M_{t}^{k}\right)=0 \quad \text { and } \quad \mathbb{E}^{x}\left(M_{t}^{k} M_{t}^{\ell}\right)=2 \mathbb{E}^{x}\left(\int_{0}^{t} a_{k \ell}\left(X_{s}\right) d s\right)
$$


for $t \geq 0$ and $x \in \bar{G}$. The processes $M_{t}^{k}$ are continuous martingales (under $\mathbb{P}^{x}$ ) with co-variation

$$
\left\langle M^{k}, M^{\ell}\right\rangle_{t}=2 \int_{0}^{t} a_{k \ell}\left(X_{s}\right) d s, \quad \text { almost surely }\left(\mathbb{P}^{x}\right),
$$

for all $x \in \bar{G}$. Moreover, $L_{t}$ is a unique positive continuous additive functional in the strict sense with Revuz measure $\sigma$ and supported by $\partial G$ and one has

$$
L_{t}=\int_{0}^{t} \mathbf{1}_{\partial G}\left(X_{s}\right) d L_{s} .
$$

Let $G \subset \mathbb{R}^{n}$ and $L(x, D)$ be as above. We consider now the quadratic form $\mathcal{E}_{D}:=\mathcal{E}$ on the domain $H_{0}^{1}(G)$,

$$
H_{0}^{1}(G):={\overline{C_{0}^{\infty}(G)}}^{\|\cdot\|_{1}}, \quad \text { where }\|\cdot\|_{1}=\|\cdot\|_{L^{2}}+\|\nabla \cdot\|_{L^{2}}
$$

Clearly, $\left(\mathcal{E}_{D}, H_{0}^{1}(G)\right)$ is a regular Dirichlet form and its generator $A_{D}$ has the domain

$$
D\left(A_{D}\right)=\left\{u \in H^{2}(G): \gamma u=0\right\},
$$

where $\gamma: H^{1}(G) \rightarrow H^{1 / 2}(\partial G)$ is the trace operator. As usual, $\gamma$ is the continuous extension of the map $\left.u \longmapsto u\right|_{\partial G}$ when $u \in C^{\infty}(\bar{G})$. Thus, $\gamma u=0$ means that $u$ attains 0 as boundary value. The space $H_{0}^{1}(G)$ can now be characterized by

$$
H_{0}^{1}(G)=\left\{u \in H^{1}(G): \gamma u=0\right\}
$$

(In Section 4 below, we will have a closer look at the trace operator.) The Markov process associated with the Dirichlet form $\left(\mathcal{E}_{D}, H_{0}^{1}(G)\right)$ is known to be an absorbing (elliptic) diffusion process. Since $G$ is bounded, the following Poincaré inequality holds

$$
\int_{G}|u(x)|^{2} d x \leq c_{0} \int_{G}|\nabla u(x)|^{2} d x, \quad u \in H_{0}^{1}(G) .
$$

By (1.2), (1.3) we get

$$
\lambda_{0}^{-1} \int_{G}|\nabla u(x)|^{2} d x \leq \mathcal{E}(u, u)
$$


and therefore

$$
\left(c_{0} \lambda_{0}\right)^{-1} \int_{G}|u(x)|^{2} d x \leq \mathcal{E}(u, u)
$$

This, however, means that on $H_{0}^{1}(G)$ the form $\mathcal{E}(\cdot, \cdot)$ is a scalar product which is equivalent to the canonical one $(\cdot, \cdot)_{1}$.

Let us return to the Dirichlet form $\left(\mathcal{E}, H^{1}(G)\right)$. We introduce the space

$$
\mathcal{H}^{1}(G):=\left\{u \in H^{1}(G): \mathcal{E}(u, \phi)=0 \text { for all } \phi \in C_{0}^{\infty}(G)\right\}
$$

or, equivalently,

$$
\mathcal{H}^{1}(G)=\left\{u \in H^{1}(G): \mathcal{E}(u, v)=0 \text { for all } v \in H_{0}^{1}(G)\right\} .
$$

Since $\mathcal{H}^{1}(G)$ is a closed subspace of $H^{1}(G)$ there is an orthogonal decomposition

$$
H^{1}(G)=\mathcal{H}^{1}(G) \oplus_{\mathcal{E}} H_{0}^{1}(G),
$$

and it is clear that $\mathcal{H}^{1}(G)$ consists of all solutions of the equation $L(x, D) u=0$ in $G$ such that $u$ and its first order partial derivatives belong to $L^{2}(G)$. In particular, the elements of $\mathcal{H}^{1}(G)$ are arbitrarily often differentiable on $\mathrm{G}$.

If $a_{k \ell}(x)=\delta_{k \ell},(1.9)$ is exactly the Weyl decomposition. Let us mention a special case when $n=2$ and $G=B_{1}(0)$ is the open unit disk with boundary $\partial G=S^{1}$. It is well known that one can construct a Dirichlet space $(\mathcal{C}, D(\mathcal{C}))$ on the boundary such that there is a one-toone correspondence between $(\mathcal{C}, D(\mathcal{C}))$ and the classical Dirichlet space $\left(\mathcal{E} / 2, \mathcal{H}^{1}(G)\right)$. Here

$$
\mathcal{E}(u, v)=\int_{B_{1}(0)} \nabla u(x) \cdot \nabla v(x) d x,
$$

and the form $\mathcal{C}$ is explicitly given by the Douglas integral

$$
\begin{aligned}
& \mathcal{C}(\phi, \psi) \\
= & \frac{1}{16 \pi} \int_{0}^{2 \pi} \int_{0}^{2 \pi}\left(\phi(\theta)-\phi\left(\theta^{\prime}\right)\right)\left(\psi(\theta)-\psi\left(\theta^{\prime}\right)\right) \sin ^{-2}\left(\frac{\theta-\theta^{\prime}}{2}\right) d \theta d \theta^{\prime}
\end{aligned}
$$

compare [8, pp. 12-13]. In Section 5 we will give a generalization of this result. 


\section{Subordination in the sense of Bochner.}

Definition 2.1. An arbitrarily often differentiable function $f:(0, \infty)$ $\longrightarrow \mathbb{R}$ is called Bernstein function if $f \geq 0$ and $(-1)^{n} f^{(n)} \leq 0$ hold for all $n \in \mathbb{N}$.

Bernstein functions can be fully characterized by a Lévy-Khinchin formula,

$$
f(x)=a+b x+\int_{0}^{\infty}\left(1-e^{-s x}\right) \mu(d s)
$$

with $a, b \geq 0$ and a non-negative measure $\mu$ on $(0, \infty)$ such that

$$
\int_{0}^{\infty} s(s+1)^{-1} \mu(d s)<\infty \text {. }
$$

The representation (2.1) shows that $f$ has an analytic continuation onto the complex half-plane $\operatorname{Re} z>0$ and is continuous up to the boundary. These and many other properties can be found in the monograph [3] by C. Berg and G. Forst. We will need one more fact about Bernstein functions (e.g. [3, Theorem 9.8]).

Theorem 2.2. Every convolution semigroup $\left\{\eta_{t}\right\}_{t \geq 0}$ of sub-probability measures on $[0, \infty)$ is uniquely characterized by some Bernstein function $f$, and vice versa. This correspondence is given by

$$
\int_{0}^{\infty} e^{-s x} \eta_{t}(d s)=e^{-t f(x)}
$$

Some of the most prominent Bernstein functions are the fractional powers,

$$
x^{\alpha}=\frac{\alpha}{\Gamma(1-\alpha)} \int_{0}^{\infty}\left(1-e^{-s x}\right) s^{-1-\alpha} d s, \quad x \geq 0,0<\alpha<1 .
$$

The corresponding convolution semigroup is the one-sided stable semigroup of order $\alpha$.

Definition 2.3. Let $\left\{T_{t}\right\}_{t>0}$ be a sub-Markovian semigroup on $L^{2}(X, m)$ where $X$ is a locally compact Hausdorff space and $m$ is a Borel 
measure such that $\operatorname{supp} m=X$. Denote by $\left\{\eta_{t}\right\}_{t \geq 0}$ the convolution semigroup with Bernstein function $f$. The semigroup $\left\{T_{t}^{f}\right\}_{t \geq 0}$ defined on $L^{2}(X, m)$ by the Bochner integral

$$
T_{t}^{f} u=\int_{0}^{\infty} T_{s} u \eta_{t}(d s)
$$

is called the subordinate semigroup of $\left\{T_{t}\right\}_{t \geq 0}$ with respect to $\left\{\eta_{t}\right\}_{t \geq 0}$ or with respect to $f$.

It is known that the subordinate semigroup is sub-Markovian and/ or Fellerian if the original semigroup is. A lot of results concerning the domain of the (subordinate) generator $A^{f}$ of $\left\{T_{t}^{f}\right\}_{t>0}$ and related functional calculi are known, see e.g. [11], [2], [16], [17]. In the next section we will use a characterization of $D\left(A^{\alpha^{\alpha}}\right)$ as interpolation spaces.

Assume that $\left\{T_{t}\right\}_{t \geq 0}$ is a sub-Markovian semigroup with generator $(A, D(A))$ and corresponding Dirichlet form $(\mathcal{E}, D(\mathcal{E}))$. By subordination - as above $f$ is a Bernstein function - we get the subordinate objects, $\left\{T_{t}^{f}\right\}_{t>0}$, its generator $\left(A^{f}, D\left(A^{f}\right)\right)$, and Dirichlet form $\left(\mathcal{E}^{f}, D\left(\mathcal{E}^{f}\right)\right)$. Let us assume that $f$ is a complete Bernstein function, which means that the representing measure $\mu$ in (2.1) is of the form

$$
\mu(d s)=\int_{0}^{\infty} e^{-s r} \rho(d r) d s
$$

where $\rho$ is a measure on $(0, \infty)$ such that

$$
\int_{0}^{\infty} \frac{\rho(d r)}{r(1+r)}<\infty
$$

Note that fractional powers are complete Bernstein functions. (Sometimes complete Bernstein functions are also called operator monotone functions, see E. Heinz [10]). From [16, Theorem 5.3] it follows that

$$
\|u\|_{L^{2}}^{2} \leq c \mathcal{E}(u, u) \quad \text { implies } \quad\|u\|_{L^{2}}^{2} \leq \frac{c}{f(1)} \mathcal{E}^{f}(u, u),
$$

for all $u \in D(\mathcal{E})$. The latter holds also on $D\left(\mathcal{E}^{f}\right)$, since we have the dense inclusions $D(A) \subset D(\mathcal{E})$ and $D(A) \subset D\left(\mathcal{E}^{f}\right)$.

Let us now discuss some probabilistic aspects of subordination. Denote again by $f$ a Bernstein function, by $\left\{\eta_{t}\right\}_{t \geq 0}$ the associated convolution semigroup on $[0, \infty)$, and assume that $f(0)=0$, thus $\eta_{0}=\delta_{0}$. 
We may interpret $\left\{\eta_{t}\right\}_{t \geq 0}$ as transition probabilities of a stochastic process $\left\{Y_{t}\right\}_{t \geq 0}$ with stationary and independent increments and càdlàg trajectories. Since $\eta_{0}=\delta_{0}$ and since the measures $\eta_{t}$ are supported on $[0, \infty)$, we have almost surely $Y_{0}=0$ and almost surely increasing paths $t \longmapsto Y_{t}$. The converse assertion is also true: every such process defines (uniquely) a convolution semigroup of probability measures on $[0, \infty)$. We will call $\left\{Y_{t}\right\}_{t \geq 0}$ subordinator.

Let $\left\{X_{t}, \mathfrak{F}_{t}\right\}_{t \geq 0}$ be a Markov process with Polish state space $(E, \mathfrak{B})$ and $\left\{Y_{t}\right\}_{t \geq 0}$ be a subordinator which is stochastically independent of $\left\{X_{t}\right\}_{t \geq 0}$. Then

$$
X_{t}^{f}(\omega):=X_{Y_{t}}(\omega):=X_{Y_{t}(\omega)}(\omega), \quad t \geq 0
$$

defines a new process $\left\{X_{t}^{f}\right\}_{t \geq 0}$ with filtration $\left\{\mathfrak{F}_{Y_{t}}\right\}_{t \geq 0}$. We say that $\left\{X_{t}^{f}\right\}_{t \geq 0}$ is obtained from $\left\{X_{t}\right\}_{t \geq 0}$ by subordination with respect to $\left\{Y_{t}\right\}_{t \geq 0}$ and call it subordinate process to $\left\{X_{t}\right\}_{t \geq 0}$.

Theorem 2.4. Let $\left\{X_{t}\right\}_{t \geq 0}$ be a Markov process, $\left\{T_{t}\right\}_{t \geq 0}$ the associated operator semigroup, $\left\{Y_{t}\right\}_{t>0}$ a subordinator (independent of $\left.\left\{X_{t}\right\}_{t \geq 0}\right)$, and $f$ the corresponding Bernstein function. For all Borel sets $B \in \mathfrak{B}, x \in E$, and $t \geq 0$ we have

$$
\mathbb{P}^{x}\left(X_{Y_{t}} \in B\right)=T_{t}^{f} \mathbf{1}_{B}(x)=\mathbb{P}^{x}\left(X_{t}^{f} \in B\right),
$$

where $\left\{X_{t}^{f}\right\}_{t \geq 0}$ stands for the Markov process corresponding to the subordinate semigroup $\left\{T_{t}^{f}\right\}_{t \geq 0}$.

This result can be found in [5].

\section{Subordinate Neumann and Dirichlet semigroups.}

Let us return to the situation of Section 1 and consider the Dirichlet form $\mathcal{E}$ on $H^{1}(G) \subset L^{2}(\bar{G})$ and generator $A=L(x, D)$ with domain

$$
D(A)=\left\{u \in H^{2}(G): \frac{\partial}{\partial \nu} u=0\right\}
$$

The semigroup associated with it, the Neumann semigroup, is denoted by $\left\{T_{t}\right\}_{t \geq 0}$. 
For any Bernstein function $f$ the subordinate semigroup $\left\{T_{t}^{f}\right\}_{t \geq 0}$ is again sub-Markovian. Thus, by the general theory of Dirichlet forms, there exists a corresponding Dirichlet form $\mathcal{E}^{f}$ with domain $D\left(\mathcal{E}^{f}\right)$ and generator $\left(A^{f}, D\left(A^{f}\right)\right)$. As usual,

$$
D\left(\mathcal{E}^{f}\right)=D\left(\left(-A^{f}\right)^{1 / 2}\right)
$$

and, if $f$ is written in terms of its representation (2.1), the subordinate generator $A^{f}$ is given by

$$
A^{f} u=-a u+b A u+\int_{0}^{\infty}\left(T_{s} u-u\right) \mu(d s), \quad u \in D(A)
$$

This formula is due to R. Phillips [15] and refinements thereof are, e.g., given in [11], [2], [17]. These results are, however, of an abstract nature. We want to determine $D\left(A^{f}\right)$ and $D\left(\mathcal{E}^{f}\right)$ in terms of function spaces. To do so, we will restrict ourselves to the case where $f(x)=x^{\alpha}$, $0<\alpha<1$, and write $\left\{T_{t}^{(\alpha)}\right\}_{t \geq 0}, \mathcal{E}^{(\alpha)}, A^{(\alpha)}$ instead of the clumsier $\left\{T_{t}^{\cdot^{\alpha}}\right\}_{t \geq 0}$ etc. In fact, we have to deal with fractional powers of the operator $-A$. Using complex interpolation, R. Seeley determined in [18] the domains of fractional powers of elliptic differential operators under regular boundary conditions.

For $G \in \mathbb{R}^{n}, \partial G$ smooth, and $s>0$ we define the space

$$
H^{s}(G)=\left\{\left.u\right|_{G}: u \in H^{s}\left(\mathbb{R}^{n}\right)\right\}
$$

normed by

$$
\|u\|_{H^{s}(G)}=\inf \left\{\|w\|_{s}:\left.w\right|_{G}=u \text { in } \mathcal{D}^{\prime}, w \in H^{s}\left(\mathbb{R}^{n}\right)\right\},
$$

where $H^{s}\left(\mathbb{R}^{n}\right), s \geq 0$, is the space

$$
H^{s}\left(\mathbb{R}^{n}\right)=\left\{u \in L^{2}\left(\mathbb{R}^{n}\right):\|u\|_{s}^{2}=\int_{\mathbb{R}}^{n}\left(1+|\xi|^{2}\right)^{s}|\widehat{u}(\xi)|^{2} d \xi<\infty\right\}
$$

For any $s \geq 0, H^{s}(G)$ is a Hilbert space and $C^{\infty}(\bar{G})$ is a dense subspace. Let us finally define for $s>3 / 2$

$$
H_{\{\partial / \partial \nu\}}^{s}(G):=\left\{u \in H^{s}(G): \frac{\partial}{\partial \nu} u=0\right\} .
$$


Observe that $H_{\{\partial / \partial \nu\}}^{2}(G)$ coincides with $D(A)$. Denote by $[\cdot ; \cdot]_{\alpha}$ complex interpolation between the spaces inside the brackets, see e.g. [22], [21]. It is well known that

$$
D\left(A^{(\alpha)}\right)=\left[L^{2}(G) ; D(A)\right]_{\alpha}, \quad 0<\alpha<1,
$$

holds. The following precise characterization is due to R. Seeley [18, Theorem 4.1]:

Theorem 3.1. Let $\left\{T_{t}^{(\alpha)}\right\}_{t \geq 0}, A^{(\alpha)}$, and $\mathcal{E}^{(\alpha)}$ be as above.

A) For $0<\alpha<1$ we have $D\left(\mathcal{E}^{(\alpha)}\right)=H^{\alpha}(G)$.

B) For $3 / 4<\alpha<1$ we have $D\left(A^{(\alpha)}\right)=H_{\{\partial / \partial \nu\}}^{2 \alpha}=(G)$.

C) For $0<\alpha<3 / 4$ we have $D\left(A^{(\alpha)}\right)=H^{2 \alpha}(G)$.

There is a similar result for the Dirichlet form $\left(\mathcal{E}_{D}, H_{0}^{1}(G)\right)$. Denote by $\left\{S_{t}\right\}_{t>0}$ the sub-Markovian semigroup given by this Dirichlet form. As above, let $\left\{S_{t}^{(\alpha)}\right\}_{t \geq 0}$ be the subordinate semigroup with respect to fractional powers $x^{\alpha}, 0<\alpha<1$. We will need some facts on Sobolev spaces, see [22], [20], [21] as standard references. For any $s \geq 0$ let $H_{0}^{s}(G):={\overline{C_{0}^{\infty}(G)}}^{\|\cdot\|_{s}}$. Then

$$
H^{s}(G)=H_{0}^{s}(G), \quad \text { if } 0 \leq s<\frac{1}{2} .
$$

If $s>1 / 2$, we define

$$
H_{D}^{s}(G):=\left\{u \in H^{s}(G): \gamma u=0\right\}
$$

and one has, cf. [22, p. 210],

$$
H_{D}^{s}(G)=H_{0}^{s}(G), \quad \text { if } \frac{1}{2}<s \leq 1 .
$$

Here $\gamma: H^{s}(G) \longrightarrow H^{s-1 / 2}(\partial G)$ is again the trace operator, $c f$. Section 1. We can now state the analogue of Theorem 3.1 which is also due to R. Seeley [18, Theorem 4.1].

Theorem 3.2. Let $\mathcal{E}_{D}^{(\alpha)}$ and $A_{D}^{(\alpha)}$ be the Dirichlet form and the generator associated with the sub-Markovian semigroup $\left\{S_{t}^{(\alpha)}\right\}_{t \geq 0}$. 

A) For $1 / 2<\alpha<1$ we have $D\left(\mathcal{E}_{D}^{(\alpha)}\right)=H_{D}^{\alpha}(G)$.
B) For $0<\alpha<1 / 2$, we have $D\left(\mathcal{E}_{D}^{(\alpha)}\right)=H^{\alpha}(G)$.
C) For $1 / 4<\alpha<1$ we have $D\left(A_{D}^{(\alpha)}\right)=H_{D}^{2 \alpha}(G)$.
D) For $0<\alpha<1 / 4$ we have $D\left(A_{D}^{(\alpha)}\right)=H^{2 \alpha}(G)$.

In view of (3.4)-(3.6) we may restate the above assertions in the form $D\left(\mathcal{E}_{D}^{(\alpha)}\right)=H_{0}^{\alpha}(G)$ for $0<\alpha<1$ but $\alpha \neq 1 / 2$, and $D\left(A_{D}^{(\alpha)}\right)=$ $H_{0}^{2 \alpha}(G)$ for $0<\alpha<1$ but $\alpha \neq 1 / 4$. The values $\alpha=1 / 4,1 / 2-$ and also the case $\alpha=3 / 4$ of Theorem 3.1 - must be treated separately. We will not do this here.

Recall that on $H_{0}^{1}(G)$ the form $\mathcal{E}$ satisfies Poincaré's inequality (1.7). By (2.3) we see that

$$
\|u\|_{L^{2}}^{2} \leq c_{0} \lambda_{0} \mathcal{E}^{(\alpha)}(u, u)
$$

holds for all $u \in H_{0}^{\alpha}(G)$. Thus, $\mathcal{E}^{(\alpha)}$ defines a scalar product that is equivalent to the canonical one $(\cdot, \cdot)_{\alpha}$ of $H_{0}^{\alpha}(G)$.

Suppose (just for the next few lines) that the coefficients of $L(x, D)$ are defined on the whole space $\mathbb{R}^{n}$ and that the fractional powers of this operator - i.e. acting on functions defined on $\mathbb{R}^{n}$ - are considered. One should note that, in this case, the Dirichlet problem for the fractional powers of $L(x, D)$ is different from the subordinated Dirichlet problem discussed above, see [12].

\section{A Weyl decomposition of $\left(\mathcal{E}^{(\alpha)}, H^{\alpha}(G)\right)$.}

Let $\mathcal{E}$ be the Dirichlet form (1.3) with domain $H^{1}(G)$ and generator A under Neumann boundary conditions, i.e., with domain $H_{\{\partial / \partial \nu\}}^{2}(G)$. For $0<\alpha<1$ denote by $\mathcal{E}^{(\alpha)}$ the Dirichlet form obtained by subordination with respect to the fractional powers $f_{\alpha}(x)=x^{\alpha}$; by Theorem 3.1 its form domain is the space $H^{\alpha}(G)$. The aim of this section is to show how one can get a Weyl-type decomposition of $H^{\alpha}(G)$ with respect to the Dirichlet form.

We put

$$
\mathcal{E}_{\lambda}^{(\alpha)}(u, v):=\left((\lambda-A)^{\alpha / 2} u,(\lambda-A)^{\alpha / 2} v\right)_{L^{2}}, \quad \lambda \geq 0
$$


and identify $\mathcal{E}_{0}^{(\alpha)}$ and $\mathcal{E}^{(\alpha)}$. Clearly, $\left(\mathcal{E}_{\lambda}^{(\alpha)}, H^{\alpha}(G)\right)$ is again a Dirichlet form, and for $\lambda>0$ the form $\mathcal{E}_{\lambda}^{(\alpha)}(\cdot, \cdot)$ is a scalar product, $c f$. (2.3), that is equivalent to the one on $H^{\alpha}(G)$. Moreover, the quadratic forms $\mathcal{E}_{\lambda}^{(\alpha)}(\cdot, \cdot)$ and $\left(A^{\alpha / 2} \cdot A^{\alpha / 2} \cdot\right)_{L^{2}}+\lambda(\cdot, \cdot)_{L^{2}}$ are equivalent. On the space $H_{0}^{\alpha}(G)$ this remains true even for $\mathcal{E}_{0}^{(\alpha)}(\cdot, \cdot)$, cf. (3.7).

For $0<\alpha \leq 1$ and $\lambda \geq 0$ we call the functions in

$$
\mathcal{H}_{\lambda}^{\alpha}(G):=\left\{u \in H^{\alpha}(G): \mathcal{E}_{\lambda}^{(\alpha)}(u, v)=0 \text { for all } v \in H_{0}^{\alpha}(G)\right\}
$$

$\mathcal{E}_{\lambda}^{(\alpha)}$-harmonic functions. Since $\overline{C_{0}^{\infty}(G)} \|^{\|\cdot\|_{\alpha}}=H_{0}^{\alpha}(G)$, one has also

$$
\mathcal{H}_{\lambda}^{\alpha}(G)=\left\{u \in H^{\alpha}(G): \mathcal{E}_{\lambda}^{(\alpha)}(u, \phi)=0 \text { for all } \phi \in C_{0}^{\infty}(G)\right\}
$$

We can now state the main result of this section.

Theorem 4.1. Let $\left(\mathcal{E}_{\lambda}^{(\alpha)}, H^{\alpha}(G)\right)$ be as above. For all $0<\alpha \leq 1$ and $\lambda \geq 0$ one has the orthogonal decomposition

$$
H^{\alpha}(G)=\mathcal{H}_{\lambda}^{\alpha}(G) \oplus_{\mathcal{E}_{\lambda}^{(\alpha)}} H_{0}^{\alpha}(G) .
$$

If $\alpha>1 / 2$, this decomposition is non-trivial in the sense that $\mathcal{H}_{\lambda}^{\alpha}(G) \supsetneqq$ $\{0\}$ and there is a canonical isomorphism

$$
\Pi_{\lambda}^{(\alpha)}: H^{\alpha-1 / 2}(\partial G) \longrightarrow \mathcal{H}_{\lambda}^{\alpha}(G) \text {. }
$$

Proof. We will, first of all, consider the case $0<\alpha<1 / 2$. Then $H^{\alpha}(G)=H_{0}^{\alpha}(G)$, and the condition in (4.1)

$$
\mathcal{E}_{\lambda}^{(\alpha)}(u, v)=0, \quad \text { for all } v \in H_{0}^{\alpha}(G)
$$

implies that $u \equiv 0$. This means that we cannot expect any non-trivial decomposition of type (4.2) if $\alpha<1 / 2$.

Assume now that $1 / 2<\alpha \leq 1$ - as already mentioned, the limiting case $\alpha=1 / 2$ will not be considered here. Note, however, that $\alpha=$ 1 does not play any special rôle and will always be included in the following considerations. Now $H_{0}^{\alpha}(G)$ and $\mathcal{H}_{\lambda}^{\alpha}(G)$ are closed subspaces of $H^{\alpha}(G)$, and since for all $u \in H^{\alpha}(G)$ the condition

$$
\mathcal{E}_{\lambda}^{(\alpha)}(u, v)=0, \quad \text { for all } v \in H_{0}^{\alpha}(G)
$$


implies that $u \equiv 0$, the decomposition (4.2) is orthogonal. (These considerations are still valid for $\alpha=1 / 2$.)

In order to show that $\Pi_{\lambda}^{(\alpha)}$ is an isomorphism we have to recall some properties of the trace operator $\gamma$. Again, [22, in particular Section 4.7] will be our standard reference. For $1 / 2<s<3 / 2$ we define $\gamma$ as above, cf. Section 1. Then $\gamma: H^{s}(G) \longrightarrow H^{s-1 / 2}(\partial G)$ is continuous and onto, and there exists a bounded linear operator $\tilde{\gamma}: H^{s-1 / 2}(\partial G) \longrightarrow H^{s}(G)$ such that $\gamma \circ \tilde{\gamma}=$ id on $H^{s-1 / 2}(\partial G)$. The kernel of $\gamma$, i.e., its nullspace is just $H_{0}^{s}(G)$. Thus, for any $u \in H^{s}(G), 1 / 2<s<3 / 2$, the trace $\gamma u \in H^{s-1 / 2}(\partial G)$ exists and $\gamma u=0$ implies that $u \in H_{0}^{s}(G)$. Conversely, for $\phi \in H^{s-1 / 2}(\partial G)$ there is a $u_{\phi}:=\tilde{\gamma} \phi \in H^{s}(G)$ such that $\gamma u_{\phi}=\phi$. However, the mappings are not canonical, in the sense that $\gamma u=\gamma w$ does not imply $u=w$.

Our aim is to construct a continuous, bijective linear map from $\mathcal{H}_{\lambda}^{\alpha}(G)$ to $H^{\alpha-1 / 2}(\partial G), \alpha>1 / 2$. By the results of the preceding paragraph we find for every $\phi \in H^{\alpha-1 / 2}(\partial G)$ some $f \in H^{\alpha}(G)$ such that $\gamma f=\phi$. Define a linear functional $\Lambda_{\lambda, f}^{\alpha}$ on $H^{\alpha}(G)$ by

$$
\Lambda_{\lambda, f}^{\alpha}(v):=\mathcal{E}_{\lambda}^{(\alpha)}(f, v), \quad v \in H^{\alpha}(G)
$$

By our assumptions, $\mathcal{E}_{\lambda}^{(\alpha)}(\cdot, \cdot)$ is for all $\lambda \geq 0$ a scalar product which is equivalent to $(\cdot, \cdot)_{\alpha}$ on $H_{0}^{\alpha}(G)$. An application of the Lax-Milgram theorem shows that there exists a unique element $\omega_{\lambda, f} \in H_{0}^{\alpha}(G)$ such that

$$
\mathcal{E}_{\lambda}^{(\alpha)}\left(\omega_{\lambda, f}, v\right)=\Lambda_{\lambda, f}^{\alpha}(v), \quad v \in H_{0}^{\alpha}(G),
$$

holds. We define

$$
u_{\lambda, f}:=\omega_{\lambda, f}-f
$$

Claim 1. $u_{\lambda, f}$ is contained in $\mathcal{H}_{\lambda}^{\alpha}(G)$. Indeed, for any $v \in C_{0}^{\infty}(G)$ we get

$$
\begin{aligned}
\mathcal{E}_{\lambda}^{(\alpha)}\left(u_{\lambda, f}, v\right) & =\mathcal{E}_{\lambda}^{(\alpha)}\left(\omega_{\lambda, f}, v\right)-\mathcal{E}_{\lambda}^{(\alpha)}(f, v) \\
& =\Lambda_{\lambda, f}^{\alpha}(v)-\mathcal{E}_{\lambda}^{(\alpha)}(f, v) \\
& =\mathcal{E}_{\lambda}^{(\alpha)}(f, v)-\mathcal{E}_{\lambda}^{(\alpha)}(f, v) \\
& =0
\end{aligned}
$$


Claim 2. $u_{\lambda, f}$ depends only on $\phi=\gamma f$ and the map $\phi \longmapsto u_{\lambda, \phi}:=u_{\lambda, f}$ is linear. Let $f_{1}, f_{2} \in H^{\alpha}(G)$ such that $f_{1} \neq f_{2}$ but $\gamma f_{1}=\gamma f_{2}=\phi$. Thus, $f_{1}-f_{2} \in H_{0}^{\alpha}(G)$ and each $f_{j}$ has an orthogonal decomposition

$$
f_{j}=u_{\lambda, f_{j}}+\omega_{\lambda, f_{j}}, \quad j=1,2,
$$

where $u_{\lambda, f_{j}} \in \mathcal{H}_{\lambda}^{\alpha}(G)$ and $\omega_{\lambda, f_{j}} \in H_{0}^{\alpha}(G)$. For every $v \in H_{0}^{\alpha}(G)$ we get

$$
\begin{aligned}
\mathcal{E}_{\lambda}^{(\alpha)}\left(f_{1}-f_{2}, v\right) & =\mathcal{E}_{\lambda}^{(\alpha)}\left(u_{\lambda, f_{1}}-u_{\lambda, f_{2}}, v\right)+\mathcal{E}_{\lambda}^{(\alpha)}\left(\omega_{\lambda, f_{1}}-\omega_{\lambda, f_{2}}, v\right) \\
& =\mathcal{E}_{\lambda}^{(\alpha)}\left(\omega_{\lambda, f_{1}}-\omega_{\lambda, f_{2}}, v\right)
\end{aligned}
$$

Since $f_{1}-f_{2} \in H_{0}^{\alpha}(G)$ and $\omega_{\lambda, f_{1}}-\omega_{\lambda, f_{2}} \in H_{0}^{\alpha}(G)$, we find $f_{1}-f_{2}=$ $\omega_{\lambda, f_{1}}-\omega_{\lambda, f_{2}}$, hence $u_{\lambda, f_{1}}=u_{\lambda, f_{2}}$. The linearity of $\phi \longrightarrow u_{\lambda, \phi}$ is obvious.

We have seen so far, that

$$
\Pi_{\lambda}^{(\alpha)}: H^{\alpha-1 / 2}(\partial G) \longrightarrow \mathcal{H}_{\lambda}^{\alpha}(G), \quad \phi \longmapsto u_{\lambda, \phi},
$$

is a well-defined linear operator.

Claim 3. The mapping $\Pi_{\lambda}^{(\alpha)}$ is bijective. Suppose that $\Pi_{\lambda}^{(\alpha)}(\phi)=0$ for some $\phi \in H^{\alpha-1 / 2}(\partial G)$. But $0=\Pi_{\lambda}^{(\alpha)}(\phi) \in H_{0}^{\alpha}(G)$, thus $\phi=0$, i.e. $\Pi_{\lambda}^{(\alpha)}$ is injective.

In order to see surjectivity, choose any $u \in \mathcal{H}_{\lambda}^{\alpha}(G) \subset H^{\alpha}(G)$ and observe that there is a $\phi \in H^{\alpha-1 / 2}(\partial G)$ such that $\gamma u=\phi$. We can thus define $u_{\lambda, \phi}:=\Pi_{\lambda}^{(\alpha)}(\phi)$. Since $\gamma u_{\lambda, \phi}=\phi$, we find $u_{\lambda, \phi}-u \in$ $H_{0}^{\alpha}(G) \cap \mathcal{H}_{\lambda}^{\alpha}(G)$, therefore $u_{\lambda, \phi}=u$. This is but to say that $\Pi_{\lambda}^{(\alpha)}$ is onto.

Claim 4. The mapping $\Pi_{\lambda}^{(\alpha)}: H^{\alpha-1 / 2}(\partial G) \longrightarrow \mathcal{H}_{\lambda}^{\alpha}(G)$ is continuous (the Hilbert spaces are equipped with their canonical, respectively, induced canonical scalar products). Since the Hilbert space $H^{\alpha}(G)$ is the orthogonal sum of two closed subspaces, the projections

$$
\pi_{1}: H^{\alpha}(G) \longrightarrow H_{0}^{\alpha}(G) \quad \text { and } \quad \pi_{2}: H^{\alpha}(G) \longrightarrow \mathcal{H}_{\lambda}^{\alpha}(G)
$$

are orthogonal projections, hence continuous. By definition, $\tilde{\gamma}$ is also continuous, and so is the composition $\Pi_{\lambda}^{(\alpha)}=\pi_{2} \circ \tilde{\gamma}$. 


\section{An analogue of the Douglas integral.}

As in the preceding sections, $G$ denotes a bounded domain with boundary $\partial G$ which shall be a $C^{\infty}$-manifold. Let us have a closer look at the spaces $H^{\alpha-1 / 2}(\partial G), 1 / 2<\alpha \leq 1$. Following J. Wloka [25, Chapter 4.2] we can define on $H^{\alpha-1 / 2}(\partial G)$ an equivalent norm in the following way: Choose a finite cover $\left\{U_{j}\right\}_{j=1}^{M}, U_{j} \subset \partial G$, of $\partial G$ by coordinate patches, and denote by $\left\{\rho_{j}\right\}_{j=1}^{M}$ a partition of unity relative to this covering. For any $\phi \in H^{\alpha-1 / 2}(\partial G)$ we put $\phi_{j}:=\rho_{j} \phi$. Then $\|\cdot\|_{H^{\alpha-1 / 2}(\partial G)}$ is equivalent to the norm $\|\mid \cdot\| \|_{H^{\alpha-1 / 2}(\partial G)}$ which is given by

$$
\left\|\left.|\phi|\right|_{H^{\alpha-1 / 2}(\partial G)} ^{2}:=\sum_{j=1}^{M}\right\||| \phi_{j} \mid \|_{H^{\alpha-1 / 2}(\partial G)}^{2} .
$$

Here,

$$
\begin{aligned}
\|\left|\phi_{j}\right|||_{H^{\alpha-1 / 2}(\partial G)}^{2}:= & \int_{\partial G}\left|\phi_{j}(x)\right|^{2} \sigma(d x) \\
& +\int_{\partial G} \int_{\partial G} \frac{\left|\phi_{j}(x)-\phi_{j}(y)\right|^{2}}{|x-y|^{n-2+2 \alpha}} \sigma(d x) \sigma(d y),
\end{aligned}
$$

where $\sigma$ is the surface measure on $\partial G$. Let us denote by $\left(\mathcal{S}^{(\alpha)}, D\left(\mathcal{S}^{(\alpha)}\right)\right.$ $=H^{\alpha-1 / 2}(\partial G)$ the quadratic form

$$
\begin{aligned}
& \mathcal{S}^{(\alpha)}(\phi, \psi) \\
& :=\sum_{j=1}^{M} \int_{\partial G} \phi_{j}(x) \psi_{j}(x) \sigma(d x) \\
& \quad+\sum_{j=1}^{M} \int_{\partial G} \int_{\partial G} \frac{\left(\phi_{j}(x)-\phi_{j}(y)\right)\left(\psi_{j}(x)-\psi_{j}(y)\right)}{|x-y|^{n-2+2 \alpha}} \sigma(d x) \sigma(d y) .
\end{aligned}
$$

It is obvious from $(5.3)$ that $\left(\mathcal{S}^{(\alpha)}, D\left(\mathcal{S}^{(\alpha)}\right)\right)$ is a regular Dirichlet form on $L^{2}(\partial G)$. In particular, the unit contraction operator $N_{\partial G}(\phi):=$ $(0 \vee \phi) \wedge 1$, leaves the form domain $D\left(\mathcal{S}^{(\alpha)}\right)=H^{\alpha-1 / 2}(\partial G)$ invariant and operates continuously thereon, i.e.,

$$
\mathcal{S}^{(\alpha)}\left(N_{\partial G}(\phi), N_{\partial G}(\phi)\right) \leq \mathcal{S}^{(\alpha)}(\phi, \phi), \quad \phi \in D\left(\mathcal{S}^{(\alpha)}\right) .
$$


Denote by $N_{G}, N_{G}(u):=(0 \vee u) \wedge 1$, the unit contraction defined for functions $u: \bar{G} \longrightarrow \mathbb{R}$. Since $H^{\alpha}(G)$ is a Dirichlet space with respect to its canonical scalar product, we find as above that $N_{G}: H^{\alpha}(G) \longrightarrow$ $H^{\alpha}(G)$ is continuous and operates on any Dirichlet form with domain $H^{\alpha}(G)$.

Lemma 5.1. Let $\gamma$ be the trace operator and $N_{\partial G}, N_{G}$ unit contractions on $\partial G$ and $G$. For $u \in H^{\alpha}(G)$ we have

$$
\gamma\left(N_{G}(u)\right)=N_{\partial G}(\gamma u)
$$

Proof. For $h \in C(\bar{G}) \cap H^{\alpha}(G)$ the assertion (5.4) is straightforward. Since $\gamma, N_{\partial G}$, and $N_{G}$ are continuous operators, so are their compositions $\gamma \circ N_{G}: H^{\alpha}(G) \longrightarrow H^{\alpha-1 / 2}(\partial G)$ and $N_{\partial G} \circ \gamma: H^{\alpha}(G) \longrightarrow$ $H^{\alpha-1 / 2}(\partial G)$, and (5.4) follows from the density of $C(\bar{G}) \cap H^{\alpha}(G)$ in $H^{\alpha}(G)$.

Let $\mathcal{E}_{\lambda}^{(\alpha)}(\cdot, \cdot), \Pi_{\lambda}^{(\alpha)}$, and $\mathcal{H}_{\lambda}^{\alpha}(G)$ be as in the preceding section. Then

$$
\mathcal{C}_{\lambda}^{(\alpha)}(\phi, \psi):=\mathcal{E}_{\lambda}^{(\alpha)}\left(\Pi_{\lambda}^{(\alpha)}(\phi), \Pi_{\lambda}^{(\alpha)}(\psi)\right), \quad \phi, \psi \in H^{\alpha-1 / 2}(\partial G),
$$

defines on $H^{\alpha-1 / 2}(\partial G)$ a bilinear form. We know already that $\Pi_{\lambda}^{(\alpha)}$ : $H^{\alpha-1 / 2}(\partial G) \longrightarrow \mathcal{H}_{\lambda}^{\alpha}(G)$ is a linear, continuous, and bijective operator. Since $\left(\mathcal{H}_{\lambda}^{\alpha}(G),(\cdot, \cdot)_{\alpha}\right)$ is a closed subspace of $H^{\alpha}(G)$, it is itself a Hilbert space and there exist constants $c_{1}, c_{2} \geq 0$ such that

$$
c_{1}\|\phi\|_{H^{\alpha-1 / 2}(\partial G)} \leq\left\|\Pi_{\lambda}^{(\alpha)}(\phi)\right\|_{H^{\alpha}(G)} \leq c_{2}\|\phi\|_{H^{\alpha-1 / 2}(\partial G)}
$$

holds. Hence, $\mathcal{C}_{\lambda}^{(\alpha)}$ is a closed form on $H^{\alpha-1 / 2}(\partial G)$.

Theorem 5.2. The bilinear form $\left(\mathcal{C}_{\lambda}^{(\alpha)}, H^{\alpha-1 / 2}(\partial G)\right)$ is a Dirichlet form.

ProOF. $\mathcal{C}_{\lambda}^{(\alpha)}$ being a closed form, it remains to prove the contraction property for the unit contraction $N_{\partial G}$,

$$
\mathcal{C}_{\lambda}^{(\alpha)}\left(N_{\partial G}(\phi), N_{\partial G}(\phi)\right) \leq \mathcal{C}_{\lambda}^{(\alpha)}(\phi, \phi), \quad \phi \in H^{\alpha-1 / 2}(\partial G) .
$$

In order to see this, we show first that

$$
N_{G}\left(\Pi_{\lambda}^{(\alpha)}(\phi)\right)=\Pi_{\lambda}^{(\alpha)}\left(N_{\partial G}(\phi)\right)+g_{\phi}, \quad \phi \in H^{\alpha-1 / 2}(\partial G),
$$


where $\Pi_{\lambda}^{(\alpha)}\left(N_{\partial G}(\phi)\right) \in \mathcal{H}_{\lambda}^{\alpha}(G)$ and $g_{\phi} \in H_{0}^{\alpha}(G)$. Since the decomposition (5.6) is necessarily unique, it is sufficient to prove that the traces satisfy

$$
\gamma\left(N_{G}\left(\Pi_{\lambda}^{(\alpha)}(\phi)\right)\right)=\gamma\left(\Pi_{\lambda}^{(\alpha)}\left(N_{\partial G}(\phi)\right)\right),
$$

that is, since $\gamma \circ \Pi_{\lambda}^{(\alpha)}=\mathrm{id}$ on $H^{\alpha-1 / 2}(\partial G)$,

$$
\gamma\left(N_{G}\left(\Pi_{\lambda}^{(\alpha)}(\phi)\right)\right)=N_{\partial G}(\phi)
$$

This, however, is just the assertion of Lemma 5.1. Using (5.6) we find

$$
\begin{aligned}
\mathcal{C}_{\lambda}^{(\alpha)}(\phi, \phi)= & \mathcal{E}_{\lambda}^{(\alpha)}\left(\Pi_{\lambda}^{(\alpha)}(\phi), \Pi_{\lambda}^{(\alpha)}(\phi)\right) \\
\geq & \mathcal{E}_{\lambda}^{(\alpha)}\left(N_{G}\left(\Pi_{\lambda}^{(\alpha)}(\phi)\right), N_{G}\left(\Pi_{\lambda}^{(\alpha)}(\phi)\right)\right) \\
= & \mathcal{E}_{\lambda}^{(\alpha)}\left(\Pi_{\lambda}^{(\alpha)}\left(N_{\partial G}(\phi)\right), \Pi_{\lambda}^{(\alpha)}\left(N_{\partial G}(\phi)\right)\right) \\
& +2 \mathcal{E}_{\lambda}^{(\alpha)}\left(\Pi_{\lambda}^{(\alpha)}\left(N_{\partial G}(\phi)\right), g_{\phi}\right)+\mathcal{E}_{\lambda}^{(\alpha)}\left(g_{\phi}, g_{\phi}\right) \\
\geq & \mathcal{C}_{\lambda}^{(\alpha)}\left(N_{\partial G}(\phi), N_{\partial G}(\phi)\right),
\end{aligned}
$$

and we are done.

Let us return to the Dirichlet form $\left(\mathcal{S}^{(\alpha)}, H^{\alpha-1 / 2}(\partial G)\right)$. Since $\mathcal{S}^{(\alpha)}$ is a closed form on $H^{\alpha-1 / 2}(\partial G), \mathcal{S}_{\mu}^{(\alpha)}(\cdot, \cdot):=\mathcal{S}^{(\alpha)}(\cdot, \cdot)+\mu(\cdot, \cdot)_{L^{2}}$ is for any $\mu>0$ a scalar product which is equivalent to $(\cdot, \cdot)_{H^{\alpha-1 / 2}(\partial G)}$. Similarly, $\mathcal{C}_{\lambda, \mu}^{(\alpha)}(\cdot, \cdot):=\mathcal{C}_{\lambda}^{(\alpha)}(\cdot, \cdot)+\mu(\cdot, \cdot)_{L^{2}}$ is also a scalar product which is equivalent to $(\cdot, \cdot)_{H^{\alpha-1 / 2}(\partial G)}$, thus $\mathcal{S}_{\mu}^{(\alpha)}$ and $\mathcal{C}_{\lambda, \mu}^{(\alpha)}$ are equivalent to each other. Since both are Dirichlet forms, we can associate with each of them a Hunt process with state space $\partial G$. One may expect that the comparability of the forms carries over to the processes. Let us briefly explain this point for $L^{1}-L^{\infty}$-estimates of the semigroups

$$
\left\{T_{t}^{\mathcal{S}_{\mu}^{(\alpha)}}\right\}_{t \geq 0} \quad \text { and } \quad\left\{T_{t}^{\mathcal{C}_{\lambda, \mu}^{(\alpha)}}\right\}_{t \geq 0}
$$

It is known that on the spaces $H^{\alpha-1 / 2}(\partial G)$ a Sobolev inequality holds, that is

$$
\|u\|_{L^{p}(\partial G)} \leq c\|u\|_{H^{\alpha-1 / 2}(\partial G)}, \quad p=\frac{2(n-1)}{n-\alpha-\frac{1}{2}} .
$$


Note that $p>2$ if $1 / 2<\alpha \leq 1$. By (5.3) we get for $\mu>0$

$$
\|u\|_{L^{p}(\partial G)}^{2} \leq c \mathcal{S}_{\mu}^{(\alpha)}(u, u) \quad \text { and } \quad\|u\|_{L^{p}(\partial G)}^{2} \leq c^{\prime} \mathcal{C}_{\lambda, \mu}^{(\alpha)}(u, u) .
$$

This implies, cf. Varopoulos et al. [23], that both semigroups satisfy the estimates

$$
\left\|T_{t}^{\mathcal{S}_{\mu}^{(\alpha)}}\right\|_{L^{1}-L^{\infty}} \leq c_{\mu}^{\prime} \frac{e^{\mu t}}{t^{(2(n-1)) /(2 \alpha-1)}}
$$

and

$$
\left\|T_{t}^{\mathcal{C}_{\lambda, \mu}^{(\alpha)}}\right\|_{L^{1}-L^{\infty}} \leq c_{\mu}^{\prime \prime} \frac{e^{\mu t}}{t^{(2(n-1)) /(2 \alpha-1)}} .
$$

In this section we have constructed the boundary Dirichlet form associated with the subordinate process $\left\{X_{t}^{(\alpha)}\right\}_{t \geq 0}$ and, likewise, with the Dirichlet form $\left(\mathcal{E}^{(\alpha)}, H^{\alpha}(G)\right)$. In the case of a Brownian motion, this was first done by M. Fukushima [6], and in a rather general (but abstract) way for general regular symmetric Dirichlet forms by M. Silverstein [19]. Here, as in the whole paper, we provide explicit constructions which allow us to determine precisely the domains in terms of function spaces. This yields additional information for studying the Dirichlet forms and/or the corresponding (boundary) process.

\section{The process associated with $\mathcal{C}_{\lambda}^{(\alpha)}$.}

We will now study the stochastic process which is generated by the Dirichlet form $C_{\lambda}^{(\alpha)}$ on the boundary $\partial G$. We will closely follow the ideas of [8], in particular Chapter 6.2. Notice, however, that the process $\left\{X_{\lambda, t}\right\}_{t \geq 0}$ generated by $L(x, D)-\lambda$ under Neumann boundary conditions is a nice Feller process with smooth densities. We may, therefore, do without the exceptional sets which frequently occur within the framework of Dirichlet forms - for a discussion of this point in the general theory we refer to M. Fukushima's paper [7]. In order to avoid technical complications we will always assume $\lambda>0$. We conclude from this, that the extended Dirichlet space and the original one $\left(\mathcal{E}_{\lambda}^{(\alpha)}, H^{\alpha}(G)\right)$ coincide.

Let us begin with $\alpha=1$, i.e., the reflected diffusion process

$$
\left\{X_{\lambda, t}\right\}_{t \geq 0}
$$


(with filtration $\left\{\mathfrak{F}_{\lambda, t}\right\}_{t \geq 0}$ ) associated with the Dirichlet form

$$
\left(\mathcal{E}_{\lambda}, H^{1}(G)\right)
$$

where $\lambda>0$ and $\mathcal{E}_{\lambda}(\cdot, \cdot):=\mathcal{E}(\cdot, \cdot)+\lambda(\cdot, \cdot)_{L^{2}}$ with $\mathcal{E}$ as in (1.3). If $\partial G$ is smooth, the surface measure $\sigma$ is a smooth measure in the sense of [8, p. 80], because by (5.15) - take $\alpha=1$ - and the finiteness of $\sigma$ the surface measure $\sigma$ is even a measure of finite energy integral [8, p. 74], hence smooth. Thus, there is a unique positive continuous additive functional $\left\{L_{\lambda, t}\right\}_{t \geq 0}$ such that $\sigma$ is its Revuz measure, see [8, pp. 187188]. One can check that $\left\{L_{\lambda, t}\right\}_{t \geq 0}$ is the boundary local time, i.e.,

$$
L_{\lambda, t}=\int_{0}^{t} \mathbf{1}_{\partial G}\left(X_{\lambda, s}\right) d L_{\lambda, s}
$$

holds, and that the support of $\left\{L_{\lambda, t}\right\}_{t \geq 0}$ equals $\partial G$. Write $\left\{\tau_{\lambda, t}\right\}_{t \geq 0}$ for the generalized right-inverse of $\left\{L_{\lambda, t}\right\}_{t \geq 0}$,

$$
\tau_{\lambda, t}(\omega):=\inf \left\{s>0: L_{\lambda, s}(\omega)>t\right\} .
$$

Clearly, $\left\{\tau_{\lambda, t}\right\}_{t \geq 0}$ is a subordinator. We may now apply [8, Theorem 6.2.1].

Theorem 6.1. Let $L(x, D)$ be as before and denote by $\left\{X_{\lambda, t}, \mathfrak{F}_{\lambda, t}\right\}_{t \geq 0}$ the Feller process corresponding to the Dirichlet form $\left(\mathcal{E}_{\lambda}, H^{1}(G)\right)$. The time-changed process $\left\{X_{\lambda, \tau_{\lambda, t}}, \mathfrak{F}_{\lambda, \tau_{\lambda, t}}\right\}_{t \geq 0}$ is given by the Dirichlet form $\left(\mathcal{C}_{\lambda}, H^{1 / 2}(\partial G)\right), \lambda>0$.

This theorem implies, in particular, that the boundary process $\left\{X_{\lambda, \tau_{\lambda, t}}\right\}_{t \geq 0}$ is comparable (on the level of Dirichlet forms) with the process on $\partial G$ being associated with the form

$$
\left((\cdot, \cdot)_{H^{1 / 2}(\partial G)}+\lambda(\cdot, \cdot)_{L^{2}}, H^{1 / 2}(\partial G)\right) .
$$

The latter, however, should be thought of as a perturbation of a Cauchy process on the boundary.

Let us now discuss the subordinate processes, i.e., the processes associated with $\left(\mathcal{E}_{\lambda}^{(\alpha)}, H^{\alpha}(G)\right)$ and $\left(\mathcal{C}_{\lambda}^{(\alpha)}, H^{\alpha-1 / 2}(\partial G)\right), \alpha>1 / 2$ and $\lambda>0$. Denote by $\left\{Y_{t}^{(\alpha)}\right\}_{t \geq 0}$ a one-sided $\alpha$-stable subordinator with Bernstein function $f_{\alpha}(x)=x^{\alpha}, 0<\alpha \leq 1$. As for (2.4) we may choose a version of $\left\{Y_{t}^{(\alpha)}\right\}_{t \geq 0}$ that is independent of $\left\{X_{\lambda, t}\right\}_{t \geq 0}$. Then

$$
X_{\lambda, t}^{(\alpha)}(\omega):=X_{\lambda, Y_{t}^{(\alpha)}}(\omega):=X_{\lambda, Y_{t}^{(\alpha)}(\omega)}(\omega), \quad t \geq 0
$$


is the subordinate (reflected diffusion) process (in the sense of Section 2) given by $\left(\mathcal{E}_{\lambda}^{(\alpha)}, H^{\alpha}(G)\right)$. Its filtration is $\left\{\mathfrak{F}_{\lambda, Y_{t}^{(\alpha)}}\right\}_{t \geq 0}$.

Due to a result of St. Orey, [14, p. 123], $\partial G$ will be a zero-capacity set if and only if $\alpha<1 / 2$. Therefore, the assumption $\alpha \geq 1 / 2$ is necessary in order to obtain a smooth boundary measure $\sigma$ and, thus, a positive continuous additive functional $\left\{L_{\lambda, t}^{(\alpha)}\right\}_{t \geq 0}$ with Revuz measure $\sigma$. As above, the finiteness of $\sigma$ and (5.5) prove that for $\alpha>1 / 2$ the measure $\sigma$ is indeed smooth. Again, $L_{\lambda, t}^{(\alpha)}$ can be identified with the boundary local time for $X_{\lambda, t}^{(\alpha)}-$ i.e., (6.1) holds with some obvious changes - with support in $\partial G$, and $\left\{\tau_{\lambda, t}^{(\alpha)}\right\}_{t \geq 0}$ will be its generalized right-inverse.

Theorem 6.2. Let $\left\{X_{\lambda, t}^{(\alpha)}, \mathfrak{F}_{\lambda, Y_{t}^{(\alpha)}}\right\}_{t \geq 0}$ be the subordinate reflected diffusion process corresponding to the Dirichlet form $\left(\mathcal{E}_{\lambda}^{(\alpha)}, H^{\alpha}(G)\right)$, $\alpha>1 / 2$. The time-changed process $\left\{X_{\lambda, \tau_{\lambda, t}^{(\alpha)}}^{(\alpha)}, \mathfrak{F}_{\lambda, Y_{\bullet}^{(\alpha)} \circ \tau_{\lambda, t}^{(\alpha)}}\right\}_{t \geq 0}$ is given by the Dirichlet form $\left(\mathcal{C}_{\lambda}^{(\alpha)}, H^{\alpha-1 / 2}(\partial G)\right), \lambda>0$.

Starting with $\left(\mathcal{E}_{\lambda}, H^{1}(G)\right)$ and $\left\{X_{\lambda, t}\right\}_{t>0}$ we have, so far, constructed three new Dirichlet forms and stochastic processes.

- The associated boundary Dirichlet form/process

$$
\left(\mathcal{C}_{\lambda}, H^{1 / 2}(\partial G)\right) \quad \text { and } \quad X_{\lambda, \tau_{\lambda, t}}, \quad t \geq 0,
$$

where $\tau_{\lambda, t}$ is the generalized inverse of the boundary local time $L_{t}$ of the original process.

- The subordinate Dirichlet form/process

$$
\left(\mathcal{E}_{\lambda}^{(\alpha)}, H^{\alpha}(G)\right) \quad \text { and } \quad X_{\lambda, t}^{(\alpha)}:=X_{\lambda, Y_{t}^{(\alpha)}}, \quad t \geq 0,
$$

where $\alpha>1 / 2$ and $Y_{t}^{(\alpha)}$ is a one-sided $\alpha$-stable subordinator.

- The boundary Dirichlet form/process associated with the subordinate form/process

$$
\left(\mathcal{C}_{\lambda}^{(\alpha)}, H^{\alpha-1 / 2}(\partial G)\right) \quad \text { and } \quad X_{\lambda, \tau_{\lambda, t}^{(\alpha)}}^{(\alpha)}:=X_{\lambda, Y_{\bullet}^{(\alpha)} \circ \tau_{\lambda, t}^{(\alpha)}}, \quad t \geq 0
$$

where $\alpha>1 / 2$ and $\tau_{\lambda, t}^{(\alpha)}$ is the generalized inverse of the boundary local time $L_{\lambda, t}^{(\alpha)}$ of $X_{\lambda, t}^{(\alpha)}$. 
It is natural to ask, whether the boundary process $X_{\lambda, \tau_{\lambda, t}^{(\alpha)}}^{(\alpha)}$ of the subordinate process can be directly obtained as subordinate process to $X_{\lambda, \tau_{\lambda, t}}$, i.e., to the boundary process of the original process. A partial answer to this question is given below.

Theorem 6.3. Let $L_{\lambda, t}, \tau_{\lambda, t}, L_{\lambda, t}^{(\alpha)}, \tau_{\lambda, t}^{(\alpha)}$, and $Y_{t}^{(\alpha)}$ be as above. Denote by $\left\{X_{\lambda, \tau_{\lambda, t}}\right\}_{t \geq 0}$ and $\left\{X_{\lambda, \tau_{\lambda, t}^{(\alpha)}}^{(\alpha)}\right\}_{t \geq 0}$ the boundary processes induced by the Dirichlet forms $\left(\mathcal{C}_{\lambda}, H^{1 / 2}(\partial G)\right)$ and $\left(\mathcal{C}_{\lambda}^{(\alpha)}, H^{\alpha-1 / 2}(\partial G)\right), \alpha>1 / 2$. Then

$$
\rho_{\lambda, t}:=L_{\lambda, \bullet} \circ Y_{\bullet}^{(\alpha)} \circ \tau_{\lambda, t}^{(\alpha)}, \quad t \geq 0
$$

defines a time-change for the process $\left\{X_{\lambda, \tau_{\lambda, t}}\right\}_{t \geq 0}$, and we have

$$
\tau_{\lambda, \bullet} \circ \rho_{\lambda, t}=Y_{\bullet}^{(\alpha)} \circ \tau_{\lambda, t}^{(\alpha)} \quad \text { and } \quad X_{\lambda, \tau_{\lambda, t}^{(\alpha)}}^{(\alpha)}=X_{\lambda, \tau_{\lambda, \bullet} \circ \rho_{\lambda, t}},
$$

i.e., the boundary process of the subordinate process can be represented as time-changed boundary process of the original process.

Proof. Clearly, the process $\rho_{\lambda, t}$ is an almost surely positive, increasing càdlàg process such that $\rho_{\lambda, 0}=0$ almost surely. Note that $Y_{\bullet}^{(\alpha)} \circ \tau_{\lambda, t}^{(\alpha)}$ is an $\mathfrak{F}_{\lambda, t}$-stopping time. Once (6.5) is established, we see from

$$
\left\{\rho_{\lambda, t}<s\right\}=\left\{\tau_{\lambda, \bullet} \circ \rho_{\lambda, t}<\tau_{\lambda, s}\right\}=\left\{Y_{\bullet}^{(\alpha)} \circ \tau_{\lambda, t}^{(\alpha)}<\tau_{\lambda, s}\right\} \in \mathfrak{F}_{\lambda, \tau_{\lambda, s}}
$$

where $s>0$, that $\left\{\rho_{\lambda, t}\right\}_{t \geq 0}$ is a family of $\mathfrak{F}_{\lambda, \tau_{\lambda, t}}$-stopping times, hence a time-change.

It is therefore enough to prove (6.5). Since $\tau_{\lambda, t}$ is a right-inverse, we have always $L_{\lambda, \bullet} \circ \tau_{\lambda, t}=t$, but $\tau_{\lambda, \bullet} \circ L_{\lambda, t}=t$ holds only at increase times $t$ of $L_{\lambda, t}$. In order to check that $Y_{\bullet}^{(\alpha)} \circ \tau_{\lambda, t}^{(\alpha)}$ is almost surely an increase time of $L_{\lambda, t}$ we have to prove that

$$
X_{\lambda, Y_{\bullet}^{(\alpha)} \circ \tau_{\lambda, t}^{(\alpha)}} \in \operatorname{supp}\left\{L_{\lambda, t}\right\}:=\left\{x \in \bar{G}: \mathbb{P}^{x}\left(\tau_{\lambda, 0}=0\right)=1\right\}
$$

For any $\tilde{\omega} \in \Omega$ and $t>0$

$$
\mathbb{P}^{X{ }_{\lambda, Y}^{(\alpha)}{ }^{(\alpha)}(\alpha)(\tilde{\omega})}\left(\tau_{\lambda, 0}^{(\alpha)}=0\right)
$$




$$
\begin{aligned}
& =\mathbb{P}\left(\theta_{Y_{\bullet}^{(\alpha)} \circ \tau_{\lambda, t}^{(\alpha)}}^{-1}\left\{\tau_{\lambda, 0}^{(\alpha)}=0\right\} \mid \mathfrak{F}_{\lambda, Y_{\bullet}^{(\alpha)} \circ \tau_{\lambda, t}^{(\alpha)}+}\right) \\
& \left.=\mathbb{P}\left(\inf \left\{s>0: L_{\lambda, s+\tau_{\lambda, t}^{(\alpha)}}^{(\alpha)}>L_{\lambda, \tau_{\lambda, t}^{(\alpha)}}^{(\alpha)}\right\}=0\right) \mid \mathfrak{F}_{\lambda, Y_{\bullet}^{(\alpha)} \circ \tau_{\lambda, t}^{(\alpha)}+}\right) \\
& =\mathbb{P}\left(\inf \left\{s>0: L_{\lambda, s+\tau_{\lambda, t}^{(\alpha)}}^{(\alpha)}>t\right\}=0 \mid \mathfrak{F}_{\lambda, Y_{\bullet}^{(\alpha)} \circ \tau_{\lambda, t}^{(\alpha)}+}\right) \\
& =1
\end{aligned}
$$

since $\tau_{\lambda, t}^{(\alpha)}$ is by its definition the right endpoint of every interval of constancy of $L_{\lambda, t}^{(\alpha)}$. We have thus seen that up to an exceptional (i.e. capacity zero) set, say $N_{1}$,

$$
X_{\lambda, Y_{\bullet}^{(\alpha)} \circ \tau_{\lambda, t}^{(\alpha)}} \in \operatorname{supp}\left\{L_{\lambda, t}^{(\alpha)}\right\}
$$

Since $\operatorname{supp}\left\{L_{\lambda, t}^{(\alpha)}\right\}$ is a quasi-support of the Revuz measure $\sigma, c f$. [8, Theorem 5.1.5], we have $\operatorname{supp}\left\{L_{\lambda, t}^{(\alpha)}\right\}=\partial G$ up to another exceptional set, $N_{2}^{(\alpha)}$, say. Thus,

$$
X_{\lambda, Y_{\bullet}^{(\alpha)} \circ \tau_{\lambda, t}^{(\alpha)}} \in \operatorname{supp}\left\{L_{\lambda, t}\right\} \cup N_{1} \cup N_{2}^{(\alpha)} \cup N_{2}^{(1)}
$$

The set $N=N_{1} \cup N_{2}^{(\alpha)} \cup N_{2}^{(1)}$ is again exceptional and, under our smoothness assumptions, even polar with respect to $\left\{X_{\lambda, t}^{(\alpha)}\right\}_{t \geq 0}$, see $[8$, Theorem 4.1.2]. Therefore we have for all $t>0$ and $x \in \bar{G}$

$$
\mathbb{P}^{x}\left(X_{\lambda, \tau_{\lambda, t}^{(\alpha)}}^{(\alpha)} \in N\right)=\mathbb{E}^{x}\left(\mathbf{1}_{N}\left(X_{\lambda, \tau_{\lambda, t}^{(\alpha)}}^{(\alpha)}\right)\right) \leq \mathbb{E}^{x}\left(\sup _{s>0} \mathbf{1}_{N}\left(X_{\lambda, s}^{(\alpha)}\right)\right)=0 .
$$

Consequently, $X_{\lambda, Y_{\bullet}^{(\alpha)} \circ \tau_{\lambda, t}^{(\alpha)}} \in \operatorname{supp}\left\{L_{\lambda, t}\right\}$ holds almost surely $\left(\mathbb{P}^{x}\right)$ for every $x$, and (6.5) follows.

In general, it seems to be wrong that the boundary process of a subordinate process is some subordinate to the boundary process of the original process, since, in general, $\rho_{\lambda, t}$ is neither a Lévy process nor an independent process. 


\section{The subordinate reflected diffusion process.}

Let $\left\{X_{t}\right\}_{t \geq 0}$ be the reflected diffusion considered in Section 1 above. Recall that the corresponding Dirichlet space is $\left(\mathcal{E}, H^{1}(G)\right)$ where $H^{1}(G) \subset L^{2}(\bar{G})$, and that one has the Skorokhod representation (1.4),

$$
\begin{aligned}
X_{t}^{k}-X_{0}^{k}= & M_{t}^{k}+\sum_{\ell=1}^{n} \int_{0}^{t} \frac{\partial a_{k \ell}}{\partial x_{\ell}}\left(X_{s}\right) d s \\
& +\sum_{\ell=1}^{n} \int_{0}^{t} a_{k \ell}\left(X_{s}\right) \nu_{\ell}\left(X_{s}\right) d L_{s} .
\end{aligned}
$$

As in the preceding section, let $\left\{Y_{t}^{(\alpha)}\right\}_{t \geq 0}$ denote an $\alpha$-stable subordinator and $X_{t}^{(\alpha)}=X_{0, t}^{(\alpha)}$ the subordinate reflected diffusion.

Theorem 7.1. Let $\left\{X_{t}^{(\alpha)}\right\}_{t \geq 0}$ be the process that is obtained from the reflected diffusion $\left\{X_{t}\right\}_{t \geq 0}$ through subordination with respect to a one-sided stable subordinator $\left\{Y_{t}^{(\alpha)}\right\}_{t \geq 0}$ of order $\alpha \in(0,1]$. Then the following Skorokhod representation holds

$$
\begin{aligned}
& \left(X_{t}^{(\alpha)}\right)^{k}-\left(X_{0}^{(\alpha)}\right)^{k} \\
& =\left(N_{t}^{(\alpha)}\right)^{k}+\sum_{\ell=1}^{n} \sum_{r \leq t} \int_{0}^{1} \frac{\partial a_{k \ell}}{\partial x_{\ell}}\left(X_{Y_{r-}^{(\alpha)}+s \Delta Y_{r}^{(\alpha)}}\right) d_{s}\left(Y_{r-}^{(\alpha)}+s \Delta Y_{r}^{(\alpha)}\right) \\
& (7.2) \quad \\
& \quad+\sum_{\ell=1}^{n} \sum_{r \leq t} \int_{0}^{1} a_{k \ell}\left(X_{Y_{r-}^{(\alpha)}}+s \Delta Y_{r}^{(\alpha)}\right) \nu_{\ell}\left(X_{Y_{r-}^{(\alpha)}+s \Delta Y_{r}^{(\alpha)}}\right) d_{s} L_{Y_{r-}^{(\alpha)}}+s \Delta Y_{r}^{(\alpha)}
\end{aligned}
$$

where $N_{t}^{(\alpha)}=M_{Y_{t}^{(\alpha)}}$ is a pure jump martingale (with respect to the time-changed filtration), $M_{t}$ is the continuous martingale part of the Skorokhod representation of $\left\{X_{t}\right\}_{t \geq 0}$, and $L_{t}$ is the boundary local time of the diffusion $\left\{X_{t}\right\}_{t \geq 0}$.

Proof. In order to keep notation to a minimum, we will sometimes omit the superscripts ${ }^{(\alpha)}$. A change of time in (7.1) with respect to the subordinator $\left\{Y_{t}\right\}_{t \geq 0}$ yields

$$
\begin{aligned}
& \left(X_{t}^{(\alpha)}\right)^{k}-\left(X_{0}^{(\alpha)}\right)^{k} \\
& \quad=M_{Y_{t}}^{k}+\sum_{\ell=1}^{n} \int_{0}^{Y_{t}} \frac{\partial a_{k \ell}}{\partial x_{\ell}}\left(X_{s}\right) d s+\sum_{\ell=1}^{n} \int_{0}^{Y_{t}} a_{k \ell}\left(X_{s}\right) \nu_{\ell}\left(X_{s}\right) d L_{s},
\end{aligned}
$$


where $M_{Y_{t}}, t \geq 0$, is just the subordinate to the continuous martingale $M_{t}$ in the Skorokhod representation of $\left\{X_{t}\right\}_{t \geq 0}$, see (1.5), (1.6). It is obvious that $M_{Y_{t}}$ is again a martingale (with respect to the timechanged natural filtration of $\left\{X_{t}\right\}_{t \geq 0}$ ) and that it is of pure jump type (since the subordinator is of this type).

In order to study the integral expressions in the above formula, we need a change-of-variable formula for Stieltjes integrals. Recall that $\left\{X_{t}\right\}_{t \geq 0}$ is a continuous process and that $t \longmapsto L_{t}(\omega)$ is a continuous, almost surely increasing function. The main difficulty is that $Y_{t}$ may have almost surely countably many jumps in finite time. By a wellknown approximation technique for Lévy processes $-c f$. L. Breiman [4, Theorem 14.27 and Proposition 8.36] - we can approximate $Y_{t}$ by processes $Y_{t}^{\varepsilon}$ whose paths are almost surely step functions with finitely many jumps in finite time,

$$
\lim _{\varepsilon \rightarrow 0} Y_{t}^{\varepsilon}(\omega)=Y_{t}(\omega), \quad \text { almost surely }\left(\mathbb{P}^{0}\right) .
$$

$\left(Y_{t}^{\varepsilon}\right.$ can be chosen to be the subordinator with

$$
c_{\alpha} \int_{\varepsilon}^{\infty}\left(1-e^{-x \xi}\right) x^{-1-\alpha} d x \longrightarrow \xi^{\alpha}, \quad \varepsilon \longrightarrow 0
$$

as characteristic exponent.) Therefore,

$$
\lim _{\varepsilon \rightarrow 0} \int_{0}^{Y_{t}^{\varepsilon}} \frac{\partial a_{k \ell}}{\partial x_{\ell}}\left(X_{s}\right) d s=\int_{0}^{Y_{t}} \frac{\partial a_{k \ell}}{\partial x_{\ell}}\left(X_{s}\right) d s,
$$

almost surely $\left(\mathbb{P}^{x}\right)$ and

$$
\lim _{\varepsilon \rightarrow 0} \int_{0}^{Y_{t}^{\varepsilon}} a_{k \ell}\left(X_{s}\right) \nu_{\ell}\left(X_{s}\right) d L_{s}=\int_{0}^{Y_{t}} a_{k \ell}\left(X_{s}\right) \nu_{\ell}\left(X_{s}\right) d L_{s}
$$

almost surely $\left(\mathbb{P}^{x}\right)$.

Assume that $s \longmapsto A_{s}(\omega)$ is a function which is for almost all $\omega$ continuous and increasing - this includes, in particular, the functions $s \longmapsto s$ and $s \longmapsto L_{s}(\omega)$ of (7.3), (7.4). We consider the pathwise defined Stieltjes integral

$$
\int_{0}^{Y_{t}^{\varepsilon}(\omega)} u(\omega, s) d A_{s}(\omega)
$$


for those $\omega$ where $s \longmapsto A_{s}(\omega)$ is continuous and increasing; $u(\omega, \cdot)$ is any continuous function. For fixed $\omega$ and $t$ we may assume (if necessary, we remove another negligible $\omega$-set) that the function $s \longmapsto Y_{s}^{\varepsilon}$ has only finitely many jumps $\sigma_{\ell}^{\varepsilon}, \ell=1,2, \ldots, k^{\varepsilon}(\omega)$, on $[0, t]$. Thus,

$$
\int_{0}^{Y_{t}^{\epsilon}(\omega)} u(\omega, s) d A_{s}(\omega)=\sum_{\ell=1}^{k^{\varepsilon}(\omega)} \int_{Y_{\sigma_{\ell}-}^{\epsilon}(\omega)}^{Y_{\sigma_{\ell}^{\epsilon}}^{\epsilon}(\omega)} u(\omega, s) d A_{s}(\omega)
$$

where $Y_{\sigma_{\ell}^{\epsilon}-}^{\epsilon}(\omega)=\lim _{r \uparrow \sigma_{\ell}^{\varepsilon}(\omega)} Y_{r}^{\epsilon}(\omega)$ denotes the left limit. In an appendix we will prove the following technical Lemma.

Lemma 7.2. Denote by $\Delta Y_{r}^{\epsilon}=Y_{r}^{\epsilon}-Y_{r-}^{\epsilon}$. Then

$$
\begin{aligned}
\int_{Y_{\sigma_{\ell}-}^{\epsilon}(\omega)}^{Y_{\sigma_{\ell}^{\epsilon}}^{\epsilon}(\omega)} u(\omega, s) d A_{s}(\omega) & \\
& =\int_{0}^{1} u\left(\omega, Y_{\sigma_{\ell}^{\epsilon}-}^{\epsilon}+s \Delta Y_{\sigma_{\ell}^{\epsilon}}^{\epsilon}\right) d_{s} A_{Y_{\sigma_{\ell}-}^{\epsilon}+s \Delta Y_{\sigma_{\ell}^{\epsilon}}^{\epsilon}}(\omega)
\end{aligned}
$$

An application of Lemma 7.2 shows

$$
\begin{aligned}
& \int_{0}^{Y(\omega)} u(\omega, s) d A_{s}(\omega) \\
& =\lim _{\varepsilon \rightarrow 0} \sum_{\ell=1}^{k^{\varepsilon}(\omega)} \int_{0}^{1} u\left(\omega, Y_{\sigma_{\ell}^{\epsilon}-}^{\epsilon}+s \Delta Y_{\sigma_{\ell}^{\epsilon}}^{\epsilon}\right) d_{s} A_{Y_{\sigma_{\ell} \epsilon^{\epsilon}}^{\epsilon}+s \Delta Y_{\sigma_{\ell}}^{\epsilon}}(\omega) .
\end{aligned}
$$

Since $\left\{Y_{t}^{\varepsilon}\right\}_{t \geq 0}$ is a pure jump process, we obtain

$$
\begin{aligned}
\sum_{\ell=1}^{k^{\varepsilon}(\omega)} \int_{0}^{1} u\left(\omega, Y_{\sigma_{\ell}^{\epsilon}-}^{\epsilon}+\right. & \left.s \Delta Y_{\sigma_{\ell}^{\epsilon}}^{\epsilon}\right) d_{s} A_{Y_{\sigma_{\ell}^{\epsilon}-}^{\epsilon}+s \Delta Y_{\sigma_{\ell}^{\epsilon}}^{\epsilon}}(\omega) \\
& =\sum_{r \leq t} \int_{0}^{1} u\left(\omega, Y_{r-}^{\epsilon}+s \Delta Y_{r}^{\epsilon}\right) d_{s} A_{Y_{r-}^{\epsilon}+s \Delta Y_{r}^{\epsilon}}(\omega)
\end{aligned}
$$

and the proof of Theorem 7.1 is finished by the following lemma. 


\section{Lemma 7.3.}

$$
\begin{aligned}
\lim _{\varepsilon \rightarrow 0} \sum_{r \leq t} \int_{0}^{1} u\left(\omega, Y_{r-}^{\epsilon}+s \Delta Y_{r}^{\epsilon}\right) d_{s} A_{Y_{r-}^{\epsilon}+s \Delta Y_{r}^{\epsilon}}(\omega) \\
=\sum_{r \leq t} \int_{0}^{1} u\left(\omega, Y_{r-}+s \Delta Y_{r}\right) d_{s} A_{Y_{r-}+s \Delta Y_{r}}(\omega) .
\end{aligned}
$$

Proof of Lemma 7.3. We fix $r \in(0, t]$ and set

$$
\begin{aligned}
v(s, \varepsilon) & :=u\left(\omega, Y_{r-}^{\epsilon}+s \Delta Y_{r}^{\epsilon}\right), \\
a_{s}^{\varepsilon} & :=A_{Y_{r-}^{\epsilon}+s \Delta Y_{r}^{\epsilon}}, \\
v(s) & :=u\left(\omega, Y_{r-}+s \Delta Y_{r}\right),
\end{aligned}
$$

and

$$
a_{s}:=A_{Y_{r-}+s \Delta Y_{r}}
$$

Then

$$
\begin{aligned}
& \left|\int_{0}^{1} v(s, \varepsilon) d a_{s}^{\varepsilon}-\int_{0}^{1} v(s) d a_{s}\right| \\
& \leq\left|\int_{0}^{1} v(s, \varepsilon) d a_{s}^{\varepsilon}-\int_{0}^{1} v(s, \varepsilon) d a_{s}\right|+\left|\int_{0}^{1} v(s, \varepsilon) d a_{s}-\int_{0}^{1} v(s) d a_{s}\right| \\
& \leq \sup _{\xi \leq Y_{t}(\omega)}|u(\omega, \xi)| \int_{0}^{1} d\left(a_{s}-a_{s}^{\varepsilon}\right)+\int_{0}^{1}|v(s, \varepsilon)-v(s)| d a_{s},
\end{aligned}
$$

where we have used the fact that $\Delta Y_{s}^{\varepsilon}(\omega) \leq \Delta Y_{s}(\omega)$, hence $a_{s}^{\varepsilon} \leq a_{s}$. Since $s \longmapsto u(\omega, s)$ is continuous, the second integral tends to 0 as $\varepsilon \longrightarrow 0$. The first integral tends also to 0 as $\varepsilon \longrightarrow 0$, because $a_{s}^{\varepsilon}$ increases to the continuous function $a_{s}$, hence, by Dini's theorem, this convergence is uniform. Therefore,

$$
\begin{aligned}
\lim _{\varepsilon \rightarrow 0} \int_{0}^{1} u\left(\omega, Y_{r-}^{\epsilon}+s \Delta Y_{r}^{\epsilon}\right) & d_{s} A_{Y_{r-}^{\epsilon}+s \Delta Y_{r}^{\epsilon}}(\omega) \\
= & \int_{0}^{1} u\left(\omega, Y_{r-}+s \Delta Y_{r}\right) d_{s} A_{Y_{r-}+s \Delta Y_{r}}(\omega) .
\end{aligned}
$$


Since

$$
\begin{aligned}
& \int_{0}^{1} u\left(\omega, Y_{r-}^{\epsilon}+s \Delta Y_{r}^{\epsilon}\right) d_{s} A_{Y_{r-}^{\epsilon}+s \Delta Y_{r}^{\epsilon}}(\omega) \\
& \leq \sup _{\xi \leq Y_{t}(\omega)}|u(\omega, \xi)| \int_{0}^{1} d_{s} A_{Y_{r-}+s \Delta Y_{r}}(\omega),
\end{aligned}
$$

and since

$$
\sum_{r \leq t} \int_{0}^{1} d_{s} A_{Y_{r-}+s \Delta Y_{r}}(\omega)=\sum_{r \leq t}\left(A_{Y_{r}}-A_{Y_{r-}}\right)<\infty,
$$

we may invoke Lebesgue's dominated convergence theorem which enables us to interchange the limit $\varepsilon \longrightarrow 0$ and the summation on $r \leq t$ on the left hand side of (7.6). This finally shows Lemma 7.3 and also Theorem 7.1.

\section{Concluding remarks.}

Many of our results do extend in an obvious way to subordination with respect to the larger class of complete Bernstein functions ( $c f$. [16] for a definition) containing the fractional powers $f_{\alpha}(x)=x^{\alpha}$ which were considered throughout our paper. This greater generality has to be paid for by the fact that it is not possible to obtain exact characterizations of domains etc. in terms of function spaces. If, however, a (complete) Bernstein function $f$ is comparable from above or below or from both sides with some fractional power $f_{\alpha}$ or $f_{\beta}$, that is, if for some $\alpha, \beta \in$ $(0,1]$ and large $x$

$$
\begin{aligned}
& f(x) \leq C f_{\alpha}(x), \\
& c f_{\beta}(x) \leq f(x),
\end{aligned}
$$

or

$$
c f_{\beta}(x) \leq f(x) \leq C f_{\alpha}(x),
$$

are satisfied, one can use some comparison result from [17] in order to identify for suitable values of $\alpha$ and $\beta$ the domains $D\left(A^{f}\right)$ or $D\left(\mathcal{E}^{f}\right)$ etc. with subspaces of $H^{\beta}(G)$ (or $H_{0}^{\beta}(G)$ ) or to prove that they contain the space $H^{\alpha}(G)$ or $H_{0}^{\alpha}(G)$. 
Furthermore, this allows us to give some rough characterization of the Dirichlet form for the corresponding boundary process - provided it exists, i.e., $\beta>1 / 2$.

\section{A. Appendix.}

We will give here the proof of Lemma 7.2. To keep notation to a minimum we will write $Y(t), A(t), \sigma_{\ell}, \ldots$ instead of $Y_{t}^{\varepsilon}, A_{t}, \sigma_{\ell}^{\varepsilon}, \ldots$

It is clearly enough to check (7.5) for fixed $\omega$ and for (deterministic) indicator functions $u(s, \omega)=\mathbf{1}_{(a, b]}(s)$

$$
\text { (A.1) } \begin{aligned}
\int_{Y\left(\sigma_{\ell}-\right)}^{Y\left(\sigma_{\ell}\right)} \mathbf{1}_{(a, b]}(s) d A(s) & =\int_{0}^{\infty} \mathbf{1}_{(a, b]}(s) \mathbf{1}_{\left(Y\left(\sigma_{\ell}-\right), Y\left(\sigma_{\ell}\right)\right]} d A(s) \\
& =\left(A\left(b \wedge Y\left(\sigma_{\ell}\right)\right)-A\left(a \vee Y\left(\sigma_{\ell}-\right)\right)\right) \vee 0 .
\end{aligned}
$$

On the other hand, we have (with the convention that $(a, b]=\varnothing$ if $a \geq b$ )

$$
\begin{aligned}
& \int_{0}^{1} \mathbf{1}_{(a, b]}\left(Y\left(\sigma_{\ell}-\right)+s \Delta Y\left(\sigma_{\ell}\right)\right) d_{s} A\left(Y\left(\sigma_{\ell}-\right)+s \Delta Y\left(\sigma_{\ell}\right)\right) \\
& =\int_{0}^{1} \mathbf{1}_{\left(\left(a-Y\left(\sigma_{\ell}-\right)\right) /\left(Y\left(\sigma_{\ell}\right)-Y\left(\sigma_{\ell}-\right)\right),\left(b-Y\left(\sigma_{\ell}-\right)\right) /\left(Y\left(\sigma_{\ell}\right)-Y\left(\sigma_{\ell}-\right)\right)\right]}(s) \\
& \quad \cdot d_{s} A\left(Y\left(\sigma_{\ell}-\right)+s \Delta Y\left(\sigma_{\ell}\right)\right) \\
& =\int_{-\infty}^{\infty} \mathbf{1}_{\left(\left(a-Y\left(\sigma_{\ell}-\right)\right) /\left(Y\left(\sigma_{\ell}\right)-Y\left(\sigma_{\ell}-\right)\right) \vee 0,\left(b-Y\left(\sigma_{\ell}-\right)\right) /\left(Y\left(\sigma_{\ell}\right)-Y\left(\sigma_{\ell}-\right)\right) \wedge 1\right]}(s)
\end{aligned}
$$

$$
\begin{gathered}
\cdot d_{s} A\left(Y\left(\sigma_{\ell^{-}}\right)+s \Delta Y\left(\sigma_{\ell}\right)\right) \\
=A\left(Y\left(\sigma_{\ell^{-}}\right)+\Delta Y\left(\sigma_{\ell}\right) \frac{b-Y\left(\sigma_{\ell-}\right)}{Y\left(\sigma_{\ell}\right)-Y\left(\sigma_{\ell^{-}}\right)} \wedge 1\right) \\
-A\left(Y\left(\sigma_{\ell^{-}}\right)+\left(a-Y\left(\sigma_{\ell^{-}}\right)\right) \vee 0\right) .
\end{gathered}
$$

It remains to check $(\mathrm{A} .1)=(\mathrm{A} .2)$ for all admissible permutations of $\left(a, b, Y\left(\sigma_{\ell}-\right), Y\left(\sigma_{\ell}\right)\right)$. These are
1) $a \leq Y\left(\sigma_{\ell^{-}}\right) \leq Y\left(\sigma_{\ell}\right) \leq b$,
2) $a \leq Y\left(\sigma_{\ell^{-}}\right) \leq b \leq Y\left(\sigma_{\ell}\right)$
3) $a \leq b \leq Y\left(\sigma_{\ell^{-}}\right) \leq Y\left(\sigma_{\ell}\right)$,
4) $Y\left(\sigma_{\ell^{-}}\right) \leq a \leq b \leq Y\left(\sigma_{\ell}\right)$,
5) $Y\left(\sigma_{\ell^{-}}\right) \leq a \leq Y\left(\sigma_{\ell}\right) \leq b$,
6) $Y\left(\sigma_{\ell^{-}}\right) \leq Y\left(\sigma_{\ell}\right) \leq a \leq b$, 
and this is an elementary - but somewhat tedious - exercise.

Acknowledgements. The first named author wants to thank Professor M. Fukushima for several discussions on reflected diffusions and Skorokhod representations while visiting Osaka University. He is also indebted to Professor H. Triebel for explaining him, during an Oberwolfach conference, some results on complex interpolation and domains of fractional powers of operators under boundary conditions. The second named author thanks Professor F. Hirsch, Evry, for his kind invitation and for the good working conditions at the University of Evry. Financial support by DFG post-doctoral fellowship Schi 419/1-1 is gratefully acknowledged.

\section{References.}

[1] Agmon, S., Douglis, A., Nirenberg, L., Estimates near the boundary for solutions of elliptic partial differential equations satisfying general boundary conditions I, II. Comm. Pure Appl. Math. 12 (1959), 623727, 17 (1964), 35-92.

[2] Berg, C., Boyadzhiev, Kh., deLaubenfels, R., Generation of generators of holomorphic semigroups. J. Austral. Math. Soc. Ser. A 55 (1993), 246-269.

[3] Berg, C., Forst, G., Potential Theory on Locally Compact Abelian Groups. Springer, Ergebnisse der Mathematik und ihrer Grenzgebiete, II. Ser. Bd. 87, 1975.

[4] Breiman, L., Probability. SIAM, Classics in Applied Mathematics. Vol. 7, Philadelphia, 1992.

[5] Feller, W., An Introduction to Probability Theory and its Applications. Vol. 2. Wiley, Series in Probab. and Math. Stat., 1971.

[6] Fukushima, M., On boundary conditions for multidimensional Brownian motions with symmetric resolvent densities. J. Math. Soc. Japan 21 (1969), 58-93.

[7] Fukushima, M., On a decompositon of additive functionals in the strict sense for a symmetric Markov process. In: Ma, Z. M., Röckner, M., Yan, J. A., (Eds.). Dirichlet Forms and Stochastic Processes. Proc. Intnl. Conf. Dirichlet Forms and Stochastic Processes, Beijing 1993. de Gruyter, (1995), 155-169. 
[8] Fukushima, M., Oshima, Y., Takeda, M., Dirichlet Forms and Symmetric Markov Processes. de Gruyter, Studies in Math. Vol. 19, 1994.

[9] Fukushima, M., Tomisaki, M., Reflecting diffusions on Lipschitz domains with cusps - Analytic construction and Skorohod representation. Potential Analysis 4 (1995), 377-408.

[10] Heinz, E., Beiträge zur Störungstheorie der Spektralzerlegung. Math. Ann. 123 (1951), 415-438.

[11] Hirsch, F., Domaines d'opérateurs représentés comme intégrales de résolvantes. J. Funct. Anal. 23 (1976), 239-264.

[12] Hoh, W., Jacob, N., On the Dirichlet problem for pseudodifferential operators generating Feller semigroups. J. Funct. Anal. 137 (1996), 19-48.

[13] Lions, J. L., Magenes, E., Non-Homogeneous Boundary Value Problems and Applications. Vol. I. Springer, Grundlehren Math. Wiss. Bd. 181, 1972.

[14] Orey, St., Polar sets for processes with stationary independent increments. In: Chover, J., (Ed.). Markov Processes and Potential Theory. Proceedings of a Symposion held at the Univ. of Wisconsin, Madison, 1967. Wiley, 1970, 117-126.

[15] Phillips, R. S., On the generation of semigroups of linear operators. Pacific. J. Math. 2 (1952), 343-369.

[16] Schilling, R. L., On the domain of the generator of a subordinate semigroup. In: Král, J., et al. (Eds.). Potential Theory - ICPT 94. Proceedings Intnl. Conf. Potential Theory, Kouty (CR), 1994. de Gruyter, 1996, 449-462.

[17] Schilling, R. L., Subordination in the sense of Bochner and a related functional calculus. J. Austral. Math. Soc. Ser. A 64 (1998), 368-396.

[18] Seeley, R., Interpolation in $L^{p}$ with boundary conditions. Studia Math. 44 (1972), 47-60.

[19] Silverstein, M., Boundary theory for symmetric Markov processes. Springer, Lecture Notes Math. 516, 1976.

[20] Triebel, H., Theory of Function Spaces. Birkhäuser, Monographs in Mathematics 78, 1983.

[21] Triebel, H., Theory of Function Spaces II. Birkhäuser, Monographs in Mathematics 84, 1992.

[22] Triebel, H., Interpolation Theory, Function Spaces, Differential Operators. Joh. Ambrosius Barth, 1995.

[23] Varopoulos, N., Saloff-Coste, L., Coulhon, T., Analysis and Geometry on Groups. Cambridge University Press, Cambridge Tracts in Math. 100, 1992. 
[24] Watanabe, S., On stable processes with boundary conditions. J. Math. Soc. Japan 14 (1962), 170-198.

[25] Wloka, J., Partielle Differentialgleichungen. Teubner, Math. Leitfäden, 1982.

Recibido: 1 de julio de 1.997

Niels Jacob

Mathematisches Institut

Universität Erlangen

Bismarckstrasse 1 1/2

D-91054 Erlangen, GERMANY

jacob@informatik. unibw-muenchen.de

and

René Schilling

The Nottingham Trent University

Mathematics

Burton Street

Nottingham NG1 4BU, ENGLAND

rls@maths.ntu.ac.uk 